\title{
Advection\#Dominated Accretion and the Spectral States of Black Hole X\#Ray Binaries: Application to Nova Muscae 1991
}

\section{Citation}

Esin, Ann A., Jeffrey E. McClintock, and Ramesh Narayan. 1997. "Advection\#Dominated Accretion and the Spectral States of Black Hole X\#Ray Binaries: Application to Nova Muscae 1991." The Astrophysical Journal 489 (2): 865-89. https://doi.org/10.1086/304829.

\section{Permanent link}

http://nrs.harvard.edu/urn-3:HUL.InstRepos:41384907

\section{Terms of Use}

This article was downloaded from Harvard University's DASH repository, and is made available under the terms and conditions applicable to Other Posted Material, as set forth at http:// nrs.harvard.edu/urn-3:HUL.InstRepos:dash.current.terms-of-use\#LAA

\section{Share Your Story}

The Harvard community has made this article openly available. Please share how this access benefits you. Submit a story.

Accessibility 


\title{
Advection-Dominated Accretion and the Spectral States of Black Hole X-Ray Binaries: Application to Nova Muscae 1991
}

\author{
Ann A. Esin, Jeffrey E. McClintock, Ramesh Narayan \\ Harvard-Smithsonian Center for Astrophysics, Cambridge, MA 02138
}

\begin{abstract}
Black hole X-ray binaries (BHXBs) are known to display five distinct spectral states. In order of increasing luminosity these are the quiescent state, low state, intermediate state, high state and very high state. We present a self-consistent model of accretion flows around black holes which unifies all of these states except the very high state. The model is an extension of the following paradigm which has been applied successfully to the quiescent state. The accretion flow consists of two zones, an inner advection-dominated accretion flow (ADAF) which extends from the black hole horizon to a transition radius $r_{t r}$, and an outer thin accretion disk that is present beyond $r_{t r}$. Above the disk is a hot corona which is a continuation of the inner ADAF. The model consistently treats the dynamics of the accreting gas, the thermal balance of the ions and electrons in the two-temperature ADAF and corona, and the radiation processes that produce the observed spectrum.

At low mass accretion rates, $\dot{m} \lesssim 0.01$ (in Eddington units), the inner ADAF zone in the model radiates extremely inefficiently, and the outer thin disk is restricted to large radii $\left(r_{t r} \sim 10^{2}-10^{4}\right.$, in Schwarzschild units). The luminosity therefore is low, and this configuration is identified with the quiescent state. For $\dot{m} \gtrsim 0.01$ and up to a critical value $\dot{m}_{\text {crit }} \sim 0.08$, the radiative efficiency of the ADAF increases rapidly and the system becomes fairly luminous. The spectrum is very hard and peaks around $100 \mathrm{keV}$. This is the low state. The exact value of $\dot{m}_{\text {crit }}$ depends on the viscosity parameter $\alpha\left(\dot{m}_{\text {crit }} \sim 1.3 \alpha^{2}\right.$; the paper assumes $\alpha=0.25)$. For values of $\dot{m}>\dot{m}_{\text {crit }}$ and up to a second critical value about $10 \%$ higher, the ADAF progressively shrinks in size, the transition radius decreases, and the X-ray spectrum changes continuously from hard to soft. We identify this stage with the intermediate state. Finally, when $\dot{m}$ is sufficiently large, the inner ADAF zone disappears altogether and the thin accretion disk extends down to the marginally stable orbit. The spectrum is dominated by an ultrasoft component with a weak hard tail. This is the high state. Model spectra calculated with this unified scenario agree well with observations of the quiescent, low, intermediate and high states. Moreover, the model provides a
\end{abstract}


natural explanation for the low state to high state transition in BHXBs. We also make a tentative proposal for the very high state, but this aspect of the model is less secure.

A feature of the model is that it is essentially parameter-free. We test the model against observations of the soft X-ray transient Nova Muscae during its 1991 outburst. The model reproduces the observed lightcurves and spectra surprisingly well, and makes a number of predictions which can be tested by observations of other BHXBs.

Subject headings: accretion, accretion disks - black holes - gamma rays radiation mechanisms - X-rays: binaries, spectra

\section{Introduction}

Galactic X-ray sources are classified as black hole X-ray binaries (BHXB, see van Paradijs \& McClintock 1995; Tanaka \& Lewin 1995; Tanaka \& Shibazaki 1996 and Liang 1997 for reviews) if the binary mass function is $\gtrsim 3 M_{\odot}$ (A0620-00, V404 Cyg, Nova Muscae 1991, GRO J1655-40, GS 2000-251, Nova Ophiuchi 1997) or if there is other less direct evidence that the accreting compact object is more massive than $3 M_{\odot}$ (e.g. Cyg X-1, LMC X-3, GRO J0422+32). In addition, those systems for which there is no reliable mass estimate, but which exhibit spectral and temporal characteristics similar to the well established BHXBs, are often also included in this category (e.g. GX 339-4, 1E1740.7-2942, GRS 1915+105).

Historically, five different spectral states of BHXBs have been identified, based on the spectral shape and flux level in the 1-10 keV X-ray band (see van der Klis 1994; Nowak 1995; Tanaka \& Lewin 1995; Tanaka \& Shibazaki 1996). Low/Hard state: Systems that have a power-law spectrum with a photon index $\alpha_{N} \sim 1.5-1.9$ and an exponential cut-off around $100 \mathrm{keV}$ are referred to as being in the "low" or "hard" state. The total X-ray luminosity in this state, computed for those systems where distance and mass estimates are available, is generally below $10 \%$ of the Eddington luminosity (Nowak 1995). High/Soft state: Other systems with spectra dominated by an ultrasoft blackbody-like component with a characteristic temperature $\sim 1 \mathrm{keV}$ (Tanaka \& Shibazaki 1996) and total luminosities exceeding the low state values are said to be in the "high" or "soft" state. In addition to the thermal component a power-law tail is also seen in the spectra of these systems, although this hard component is much less luminous than in the low state. The normalization of the power-law component is highly variable, as opposed to the soft blackbody component 
which is quite stable, and the photon index of the power-law is essentially fixed at $\alpha_{N} \sim 2.5$ (Tanaka \& Shibazaki 1996). Intermediate state: Recently, a third state has been identified (Ebisawa et al. 1994, hereafter E94; Mendez \& van der Klis 1997; Belloni et al. 1996), referred to sometimes as the "intermediate state". This state is seen during transitions between the low and high states. As the name implies, the observed spectra in this state are intermediate in character between the low and high states. Very High state: Occasionally, in some systems at very high luminosities, the non-thermal tail and blackbody component become comparable in flux, giving rise to the so called "very high" state (e.g. van der Klis 1994, Gilfanov et al. 1995). The power-law component in this case has a photon index $\sim 2.5$ and does not show evidence of a cut off even out to a few hundred keV. Quiescent/Off state: Finally, in the last few years it has become possible to measure X-ray spectra of systems in the "off" or "quiescent" state. These observations have been done on a subset of BHXBs, called soft X-ray transients (SXTs), which spend most of their lives in quiescence. Quiescent spectra are distinctly non-blackbody, with photon indices somewhat softer than in the low state, and with flux levels lower by several orders of magnitude than in the other four states (McClintock, Horne, \& Remillard 1995; Narayan, Barret, \& McClintock 1997, hereafter NBM; Narayan, Garcia \& McClintock 1997).

Within the limitations imposed by the uncertainties in the distance to each particular system and the mass of the accreting black hole, the five spectral states can be arranged in the following sequence of increasing total luminosity: quiescent state, low state, intermediate state, high state, and very high state. This order seems to be preserved when individual systems undergo state transitions and also when one binary is compared to another (provided the luminosity is expressed in Eddington units). However not all BHXBs display all the states, and in fact most systems spend the bulk of their time in a single state, making only brief excursions to other states. Cygnus X-1, for instance, is generally found in the low state, with occasional high state transitions, while LMC X-3 is nearly always in the high state (Tanaka \& Lewin 1995). The transient sources (e.g. A0620-00, Nova Muscae, V404 Cyg) are the most interesting in this respect since they undergo dramatic outbursts during which their luminosities vary over many orders of magnitude. Consequently, some transients cycle through all five states. Nova Muscae 1991 (also known as GS 1124-683), for instance, went very quickly from quiescence to the very high state and then decayed via the high, the intermediate and low states back to the quiescent state (E94).

During the past two decades a great deal of observational data has been accumulated on the behavior of BHXBs. The temporal and spectral characteristics of these sources have been widely studied, but the precise nature of the different spectral states - their relationships to one another and the mechanisms driving the state transitions - remain very uncertain. The thermal radiation seen in the high and very high states is generally modeled 
as modified blackbody emission from a geometrically thin, optically-thick accretion disk extending down to the last stable orbit (Shakura \& Sunyaev 1973, Novikov \& Thorne 1973). The power-law component seen in most states is attributed to Compton upscattering of soft photons in an optically thin hot corona above the disk (e.g. Melia \& Misra 1993; Svensson \& Zdziarski 1994) or an optically thick Comptonizing cloud (Sunyaev \& Titarchuk 1980). However, no model has been able to explain the relationship between the thermal and the "non-thermal" spectral states and the cause of the transition from one to the other remains unknown. The dramatic outbursts displayed by SXTs present an even greater challenge to existing models.

Since SXT outbursts are similar in some respects to outbursts observed in dwarf novae, Mineshige \& Wheeler (1989) and Huang \& Wheeler (1989) proposed that the disk instability model developed to explain cataclysmic variables operates also in black hole systems. This model has been fairly successful in reproducing the basic features of the observed light curves and appears to capture some of the underlying physics of the outbursts in BHXBs (see Cannizzo 1993 for a review). However, Lasota, Narayan, \& Yi (1996) and Mineshige (1996) showed that the model is inconsistent with the observed recurrence time between outbursts. Moreover, since the model requires the thin disk to extend down to the last stable orbit, it cannot explain the X-ray emission observed in the spectra of quiescent SXTs (Narayan, McClintock \& Yi 1996, hereafter NMY).

During the last few years, two new accretion solutions, that go under the general name of advection-dominated accretion flows or ADAFs, have been extensively studied. ADAFs are based on the usual $\alpha$-viscosity prescription, but differ from the standard thin accretion disk model in that a substantial fraction of the viscously dissipated energy is stored in the gas and advected to the central object with the accretion flow, rather than being radiated. The two branches of ADAFs are distinguished by the physical mechanism that causes energy advection. When the mass accretion rate is very high, the flow is optically thick, so that most of the emitted photons are dragged into the black hole by the accreting gas. This regime was first considered by Katz (1977) and Begelman (1978) and studied comprehensively by Abramowicz et al. (1988). The second branch (Narayan \& Yi 1994, 1995a,b; Abramowicz et al. 1995; Chen 1995; Chen et al. 1995) occurs when the accretion rate is lower than a certain critical rate, $\dot{m}_{\text {crit }} \sim 1.3 \alpha^{2}$ in Eddington-scaled units ( $\S 3.3$, see also Rees et al. 1982). In this case, the flow is optically thin and the gas is unable to cool efficiently. The energy thus remains in the gas in the form of thermal energy of the particles and is once again advected into the black hole. In this paper we focus on the second, low- $\dot{m}$, optically thin branch of ADAFs. This branch of solutions has been shown to be thermally and viscously stable (Abramowicz et al. 1995; Narayan \& Yi 1995b), except perhaps for a weak instability associated with certain short wavelength perturbations (Kato, Abramowicz, 
\& Chen 1996)

Two key assumptions of the optically thin ADAF solutions are that (1) most of the viscous energy dissipated in the accretion process goes into heating the ions (Shapiro, Lightman \& Eardley 1976, hereafter SLE; Rees et al. 1982), and (2) energy transfer from ions to electrons is restricted to Coulomb collisions. In the low density accreting gas Coulomb transfer is inefficient, so that the gas becomes a two-temperature plasma (SLE) where the ions are nearly virial and contain most of the viscously dissipated energy. Because of the high ion temperature, pressure support is important and the accreting flow assumes a nearly spherical configuration with sub-Keplerian rotation (Narayan \& Yi 1995a). In contrast to the ions, the electrons radiate efficiently via synchrotron and bremsstrahlung emission and inverse Compton scattering. As a result the electrons are much cooler than the ions.

The optically thin ADAF solution has had significant success in explaining the quiescent states of several SXTs (see Narayan 1997 for a review). NMY and NBM proposed that the accretion flow in quiescent SXTs consists of two zones, an inner optically thin ADAF extending from the black hole horizon to a transition radius at $\sim 10^{4}$ Schwarzschild radii and an outer thin disk beyond this radius. Using this model they explained the optical and X-ray spectra of two well-studied systems, V404 Cyg and A0620-00, while Hameury et al. (1997) found a good fit for GRO J1655-40. As mentioned earlier, the observed spectra are inconsistent with any model based on a thin disk extending down to the last stable orbit. Further, Lasota et al. (1996b) showed that by limiting the thin disk to large radii the recurrence time between outbursts in the disk instability model is in better agreement with the observations. Finally, Hameury et al. (1997) demonstrated by means of detailed numerical simulations that the model reproduces well the observed optical and X-ray lightcurves of GRO J1655-45 during its recent outburst. In particular, the model naturally explains the 6-day X-ray delay observed by Orosz et al. (1997). This delay is hard to reconcile with the original version of the disk instability model as proposed by Mineshige \& Wheeler (1989).

In the NMY and NBM model of quiescent SXTs, the mass accretion rate is fairly low, of the order of $\sim 10^{-3}$ Eddington units, and the luminosity is extremely low (because of advection), of the order of $10^{-5}$ to $10^{-7}$ Eddington units. Narayan (1996) extended the model to higher mass accretion rates and argued that many of the other spectral states of BHXBs are naturally explained with the same model. The present paper develops these ideas further. We present detailed self-consistent models of accretion flows around black holes for a wide range of mass accretion rate and compute realistic emission spectra of such flows. In $\S 2$ we review the basic equations governing ADAFs, and summarize the details 
of our model. We focus mainly on various improvements introduced in this work, namely inclusion of the advection term in the electron energy equation whose importance has been emphasized by Nakamura et al. (1997), a more realistic treatment of the ADAF-disk interaction including X-ray reflection and iron fluorescent line emission, and calculations of the equilibrium pair density. In $\S 3$ we explore the properties of the model and show that as we increase the mass accretion rate and track the transition radius consistently, the model spectra we calculate naturally go through a sequence of states which resemble the quiescent state, low state, intermediate state and high state. We also propose a speculative model for the very high state. In $\S 4$ we focus on the outburst of a typical SXT, Nova Muscae 1991, and compare the results of our model with the X-ray data published by E94. We conclude with a discussion in $\S 5$ and point out some predictions of the model which can be tested with future observations.

\section{Modeling the Accretion Flow}

\subsection{Geometry of the Flow and Model Parameters}

We consider a binary system consisting of a Schwarzschild black hole of mass $M$ accreting gas from a companion star at a rate $\dot{M}=\dot{m} \dot{M}_{E d d}$, where we define the Eddington accretion rate as $\dot{M}_{E d d}=10 L_{E d d} / c^{2}=1.39 \times 10^{18}\left(M / M_{\odot}\right) \mathrm{g} \mathrm{s}^{-1}$, corresponding to a radiative efficiency of 0.1 . We take the rotation axis of the accretion flow to be inclined at an angle $i$ to the line of sight. In our model the accretion flow has two distinct zones. From the outer

radius of the flow, $r_{\text {out }}$ (hereafter all radii are in Schwarzschild units, $R_{S c h w}=2 G M / c^{2}$ ), to a transition radius, $r_{t r}$, the gas accretes via a standard thin disk with a hot corona above the disk. For $r<r_{t r}$ the entire flow is assumed to be hot and quasispherical. We model both the hot gas in the inner region and the gas in the corona above the outer thin disk as an advection-dominated accretion flow (ADAF). We make no distinction between these two regions except for the fact that the mass accretion rate in the corona is a fraction of the total $\dot{m}$.

Following NBM, we assume that $\dot{m}$ in the corona is inversely proportional to $r$, i.e.

$$
\dot{m}_{c}=\dot{m}_{c, 0}\left(\frac{r_{t r}}{r}\right), \quad r \geq r_{t r}
$$

In general we take $\dot{m}_{c, 0}=\dot{m}(\S \S 3.1-3.3)$, except when the thin disk extends all the way to the last stable orbit, in which case we allow $\dot{m}_{c, 0}$ to be less than $\dot{m}$ (see $\S \S 3.4,3.5$ ). The functional form of $\dot{m}_{c}$ in (2-1) is quite arbitrary, though it is not implausible. Our physical picture of the corona is that it is a hot accretion flow above the thin disk which is fed by 
evaporation of mass from the surface of the disk (NBM). Clearly, $\dot{m}_{c}$ must increase inward, and considering that the energy required for evaporation ultimately has to come from gravity (cf. the discussion of "siphon flow" in Meyer \& Meyer-Hofmeister 1994), a form such as (2-1) appears likely. In principle, we could make $\dot{m}_{c} \propto r^{-\zeta}$ and treat $\zeta$ as a free parameter, but it is not clear that this would lead to any further insights into the physics of the corona.

The mass $M$ of the black hole, inclination $i$ of the binary, and the outer accretion disk radius $r_{\text {out }}$ are often constrained by observations. Also the transition radius, $r_{t r}$, can be estimated from the maximum width of the $\mathrm{H}_{\alpha}$ emission line, as discussed in NMY and NBM. However, there is in general no direct estimate of the mass accretion rate $\dot{m}$, so we consider it an adjustable parameter.

Our physical model of the ADAF is very similar to that described by NBM. We use the standard viscosity prescription (Frank, King, \& Raine 1992) parameterized through the viscosity coefficient $\alpha$, and assume that the hot gas and tangled magnetic fields in the $\mathrm{ADAF}$ are roughly in equipartition, so that $p_{\text {gas }}=[\beta /(1-\beta)] p_{\text {mag }}=\beta p_{\text {tot }}$. We take $\alpha$ and $\beta$ to be independent of radius.

In the remainder of this section we summarize how we compute the physical properties of the ADAF. Many of the details are described exhaustively in NBM, so we emphasize here only the modifications and enhancements that we have introduced in the present calculations.

\subsection{Flow Dynamics and Energy Balance}

Following NBM, we model the ADAF as a set of nested spherical shells truncated near the pole to mimic the flattening of the density profile. The shells are uniformly spaced in $\log (r)$ and are centered at radii $r_{j}$. Within each shell the radial velocity $v_{j}$, angular velocity $\Omega_{j}$, isothermal sound speed $c_{s, j}$, and viscous heating rate $Q_{j}^{+}$are calculated using the global dynamical solutions described by Narayan, Kato \& Honma (1997) and Chen, Abramowicz \& Lasota (1997). Each global solution is uniquely specified by the viscosity parameter $\alpha$, the ratio of the specific heats of the accreting gas $\gamma=(8-3 \beta) /(6-3 \beta)$ (see Esin 1997), and the advection parameter $f(r)$. The last quantity is defined to be the ratio of the energy advected by the gas to the total energy dissipated in the ADAF and is in general a function of $r$, however, in calculating the dynamical solutions we replace it by a constant $f_{a v}$ as described below. The global solutions satisfy the basic conservation laws of accretion flows - conservation of mass, radial momentum, angular momentum and energy - and ensure the 
dynamical self-consistency of the models.

In previous applications of the ADAF model to BHXBs (NMY, NBM, Hameury et al. 1997), the objective was to model only the quiescent state of the systems in which $\dot{m}$ is very low. At such $\dot{m}$, the radiative efficiency is extremely low (cf. Narayan \& Yi 1995b), which implies that $f(r) \rightarrow 1$ at each radius. It was therefore possible in those studies to set $f(r)$ equal to unity without loss of self-consistency (NBM, NMY). The goals of the present paper are more ambitious. Here we attempt to explain the entire range of observed BHXB spectral states, including quite luminous systems for which $f$ is significantly less than 1 . We therefore need a more sophisticated scheme where the global solution corresponds to the actual value of $f$ as determined by the cooling. For this purpose, we characterize the ADAF by the following average advection parameter, $f_{a v}$,

$$
f_{a v}=\frac{\sum_{j} Q_{j}^{a d v}}{\sum_{j} Q_{j}^{+}}
$$

where $Q_{j}^{+}$and $Q_{j}^{a d v}$ are the viscous heating rate and the rate of energy advection in the $j^{\text {th }}$ shell respectively, and the sums are computed over all spherical shells.

In regions where the gas flow is a pure $\operatorname{ADAF}\left(r<r_{t r}\right)$, the advected energy is simply the difference between the viscous heating and total radiative cooling,

$$
Q_{j}^{a d v}=Q_{j}^{+}-Q_{j}^{-}, \quad r<r_{t r}
$$

The quantity $Q_{j}^{+}$is obtained from the global dynamical solution while $Q_{j}^{-}$is known once the radiative processes and spectrum have been calculated ( 2.3$)$. However, at radii where the hot flow is in the form of a corona above the thin disk, we need to take into account the energy required to evaporate the gas from the thin disk into the corona. The evaporation energy in shell $j$ is estimated to be the thermal energy of the gas evaporated locally, i.e.

$$
Q_{j}^{\text {evap }}=\left[\dot{M}_{c}\left(r_{j}\right)-\dot{M}_{c}\left(r_{j+1}\right)\right]\left[\frac{3}{2} \frac{k T_{i}}{\mu_{i} m_{u}}+a\left(T_{e}\right) \frac{k T_{e}}{\mu_{e} m_{u}}\right], \quad r \geq r_{t r},
$$

where $T_{i}$ and $T_{e}$ are the ion and electron temperatures in the ADAF, the coefficient $a\left(T_{e}\right)$ describes the thermal energy of the electrons (Appendix A), $\mu_{i}=1.23$ and $\mu_{e}=1.14$ are the effective molecular weights of the ions and the electrons respectively, computed for a hydrogen mass fraction of $75 \%$ (cf. Narayan \& Yi 1995b), and we have neglected the thermal energy of the gas in the thin disk. Where does the energy for evaporation come from? The uncertainties in the physical processes responsible for evaporation make it difficult to answer this question. We simply assume that the energy is supplied entirely by the hot flow, perhaps via ion and electron conduction as suggested by Meyer \& Meyer-Hofmeister (1994). 
Under this assumption, the energy advected by the gas in the accretion flow is given by

$$
Q_{j}^{a d v}=Q_{j}^{+}-Q_{j}^{-}-Q_{j}^{e v a p}, \quad r \geq r_{t r} .
$$

Equations (2-2)-(2-5) give the average advection parameter $f_{a v}$ of the flow. Using this $f_{a v}$, we compute the global solution using the method described in Narayan, Kato, \& Honma (1997). As mentioned above, this gives the values of $v_{j}, \Omega_{j}, c_{s, j}$, and $Q_{j}^{+}$in each shell $j$. The radial velocity, combined with the equation of mass conservation, allows us to compute the mass density of the gas $\rho$ at each radius, as well as the ion and electron number densities, $n_{i}$ and $n_{e}$.

To solve for the ion and electron temperatures, $T_{i}$ and $T_{e}$, in the two-temperature ADAF, we need two relations for each radial shell. One constraint is provided by the equation for the local gas pressure,

$$
P_{\text {gas }}=\beta \rho c_{s}^{2}=n_{i} k T_{i}+n_{e} k T_{e},
$$

while the second relation is given by the energy balance equation for the electrons,

$$
Q_{j}^{e, a d v}=Q_{j}^{i e}+\delta Q_{j}^{+}-Q_{j}^{-}
$$

Equation (2-7) states that the rate of energy advection by electrons (left-hand side) is equal to the rate of energy transfer from ions to electrons via Coulomb collisions, $Q_{j}^{i e}$, plus a fraction $\delta$ of the viscous dissipation, minus the radiative cooling rate, $Q_{j}^{-}$. In all our models we set $\delta=10^{-3} \sim m_{e} / m_{p}$, which means that only $10^{-3}$ of the viscous heat goes directly into the electrons. The exact value we choose for this constant is not important. We find that the results presented in this paper are practically unchanged for any value of $\delta \lesssim 10^{-2}$. In our earlier work, we ignored $Q_{j}^{e, a d v}$, assuming that it is small relative to the terms on the right. However, Nakamura et al. (1997) have recently shown that electron energy advection can be important in some cases. We therefore include this term and compute it self-consistently using the relations given in Appendix A. We find that at high $\dot{m}$ the models are essentially unaffected by the electron advection term. However, at low $\dot{m}$, this term acts as an extra energy source for the electrons, thus increasing the overall luminosity of such flows (see §3.2). Equations (2-6) and (2-7) allow us to solve for $T_{i}$ and $T_{e}$ consistently in each radial shell.

\subsection{Radiative Transfer, Reflection, and the Iron Fluorescence Line}

The rate of cooling of the accreting gas is calculated using the iterative scattering method described in detail in NBM. Given the current guess of the temperature and density 
profile, the synchrotron and bremsstrahlung emission from each shell is first computed. Then, using the probability matrix elements $P_{i j}^{a a}$ ( or $P_{i j}^{d a}$ ), which give the probability that a photon emitted in shell $i$ of the ADAF (or ring $i$ of the thin disk) is scattered by an electron in shell $j$ of the ADAF, the rate of cooling through Compton scattering is calculated (see NBM for details). The iteration method ensures that multiple scattering within the ADAF, as well as the interaction between the ADAF and the outer disk are properly taken into account. Gravitational redshift, Doppler boosts and ray deflections are neglected.

The emission from the thin disk is calculated using the standard multicolor blackbody method (cf. Frank et al. 1992). The effective temperature of the emission from ring $i$ is determined by

$$
\sigma T_{e f f, i}^{4} A_{i}=Q_{d i s k, i}^{+}+Q_{i}^{a b s}
$$

where $A_{i}$ is the surface area of ring $i$ of the thin disk, $Q_{d i s k, i}^{+}$is the viscous dissipation rate in the ring due to the local mass accretion rate in the disk, $\dot{m}_{d}=\dot{m}-\dot{m}_{c}$, and $Q_{i}^{a b s}$ is the rate at which energy is absorbed by the disk as a result of irradiation from the ADAF. The rate of irradiation is computed using another matrix $P_{i j}^{a d}$ which gives the probability that a photon emitted in shell $i$ of the ADAF is incident on ring $j$ of the thin disk.

NBM made the simplifying assumption that all the radiation incident on the thin disk is absorbed and reprocessed into blackbody radiation, ignoring the fact that a significant fraction of the irradiating flux is scattered by the material near the disk surface and effectively reflected. This is a valid approximation for modeling quiescent SXTs in which the disk is restricted to large radii so that the contribution of the disk to the observed spectrum is negligible. However, in the present work we consider a range of transition radii and need to model the reflection more carefully.

Given the incident spectrum, we compute the spectrum of the reflected radiation using the angle-averaged Green's function for Compton reflection of monoenergetic photons from an optically thick cold disk (White, Lightman \& Zdziarski 1988; Lightman \& White 1988). The Green's function, $G\left(E, E_{0}\right)$, is defined as the probability that an incident photon with initial energy $E_{0}$ emerges from the cold medium with energy $E$. Then, for the net irradiating spectrum $\sum_{j} L_{j, i}\left(E_{0}\right)$, where $L_{j, i}\left(E_{0}\right)$ is the specific luminosity incident on ring $i$ of the thin disk from ADAF shell $j$, the reflected spectrum is simply

$$
L_{i}^{\mathrm{refl}}(E)=\sum_{j} \int_{E}^{\infty} G\left(E, E_{0}\right) L_{j, i}\left(E_{0}\right) d E_{0}
$$

For photons with initial energies below $\sim 15 \mathrm{keV}$, the value of the Green's function depends on the bound-free absorption opacity of the cold material, which is a function of the ionization structure. Since the thin disk is relatively cold, its ionization state is largely 
determined by the irradiating X-ray flux, and in principle can be calculated self-consistently in our model. However, the ionization does not have a strong effect on the dominant part of the reflected spectrum between 10 and $300 \mathrm{keV}$ (Lightman \& White 1988), so for simplicity, we have used the absorption opacity for a completely neutral plasma with cosmic metal abundances (Morrison \& McCammon 1983). Having computed the reflected spectrum, the absorbed energy $Q_{i}^{a b s}$ is simply the difference between the incident and reflected luminosities

$$
Q_{i}^{a b s}=\int_{0}^{\infty}\left[\sum_{j} L_{j, i}(E)-L_{i}^{\mathrm{refl}}(E)\right] d E .
$$

Both the reflected component and the standard thin disk blackbody emission then become a part of the iterative scattering procedure. This allows us to take into account Comptonization of the disk photons by the coronal electrons.

We also model the iron $\mathrm{K} \alpha$ fluorescence line which is produced through photoelectric absorption of X-ray photons with energies above the K-shell absorption edge, $E_{K}$. Such line features have been observed in the spectra of Seyfert I galaxies (e.g. Tanaka et al. 1995; Nandra \& Pounds 1994) and in the galactic BHXB Cyg X-1 (e.g. Ebisawa et al. 1996; Gierliński et al. 1997). As in our calculations of the reflection component, we assume that the disk contains mainly neutral material with a cosmic abundance of $3.3 \times 10^{-5}$ iron atoms per hydrogen atom (Morrison \& McCammon 1983). We compute the flux in the iron line using the function $\mathcal{G}\left(E_{0}, \cos \theta_{0}\right)$ given by George \& Fabian (1991), which they defined as the fraction of ADAF photons of initial energy $E_{0}$, incident on the thin disk at an angle $\theta_{0}$, that give rise to iron fluorescence photons able to escape the disk. The resultant luminosity in the iron line from ring $i$ of the thin disk is then

$$
L_{i}^{\mathrm{Fe}}=\sum_{j} \int_{E_{K}}^{\infty} L_{j, i}\left(E_{0}\right) \mathcal{G}\left(E_{0},\left\langle\cos \theta_{0}\right\rangle_{j, i}\right) d E_{0}
$$

where $\left\langle\cos \theta_{0}\right\rangle_{j, i}$ is averaged over all photons from ADAF shell $j$ incident on ring $i$ of the thin disk, and the K-shell absorption edge is $E_{K}=7.1 \mathrm{keV}$ for neutral iron atoms (George $\&$ Fabian 1991). In our model both $L_{j, i}\left(E_{0}\right)$ and $\left\langle\cos \theta_{0}\right\rangle_{j, i}$ are completely specified for every pair of indices $j$ and $i$, so that the integral is easily computed. We do not model the K $\alpha$ line profile in detail, but simply take it to be a Gaussian centered at $6.4 \mathrm{keV}$ with a fractional Doppler width equal to $\sin i /(2 r)^{1 / 2}$, where $i$ is the disk inclination.

\subsection{Pair Production}

The electrons in the inner parts of the ADAF reach relativistic temperatures of $10^{9}-10^{10} \mathrm{~K}$ making pair production possible there, as a result of particle-particle, 
particle-photon, and photon-photon interactions. Kusunose \& Mineshige (1996) and Björnsson et al. (1996) concluded that the effect of pair production on the structure of ADAFs is small as long as $\alpha<1$. Since our models are calculated with $\alpha=0.25$, this would suggest that pair processes are not likely to be important. Nevertheless, in the interest of consistency we have included pair interactions, though with some simplifications as noted below. We confirm that pair effects are negligible for the branch of (low- $\dot{m}$ ) ADAFs studied in this paper.

In our calculations we assume that the pair fraction, defined as the ratio of the positron and proton number densities, $z=n_{+} / n_{p}$, is low. In addition, although we do include particle-photon, and photon-photon interactions in our calculations, we assume that particle-particle pair production dominates. This is valid since ADAFs produce very few photons with energy $\gtrsim m_{e} c^{2}$ needed to create pairs. These assumptions allow us to neglect the photon balance equation. Similarly, we drop the advection term in the continuity equation for pairs. This term acts as an additional sink term and reduces the equilibrium pair density; since we neglect it, our results correspond to an upper limit on $z$. With these simplifications, the problem reduces to solving just the pair balance equation,

$$
\left(\dot{n}_{+}\right)_{\text {prod }}=\left(\dot{n}_{+}\right)_{\mathrm{ann}}
$$

where the term on the left is the rate of production of pairs and that on the right is the annihilation rate. Appendix B gives detailed expressions (taken from the literature) for the various pair production and pair annihilation processes in a thermal plasma. We also show there how equation $(2-12)$ can be reduced to a quadratic equation in $z$ and solved iteratively.

Our results indicate that pair production is not a significant process in ADAFs, at least in the parameter range explored in this paper (see $\S 3$ for details); in all cases we find $z \lesssim 10^{-5}$. This result is not surprising. Even though electrons in advection-dominated flows can reach temperatures on the order $\sim m_{e} c^{2}$, the highest temperatures are achieved only at relatively low $\dot{m}$, where the optical depth and the luminosity of the flow are very low. At accretion rates near Eddington, the flow is much cooler with $T_{e} \lesssim 0.2 m_{e} c^{2}$, and the bulk of the emission is significantly below the pair production threshold. In other words, the compactness parameter $l=L_{\gamma} \sigma_{T} / R m_{e} c^{3}$, where $L_{\gamma}$ is the luminosity above $\sim 511 \mathrm{keV}$ and $R \sim 10 R_{S c h w}$ is the size of the emitting region, is always significantly below unity, ensuring that pair processes do not dominate the physics of the flow. 


\subsection{Numerical Solution}

Given the system parameters $M, i, r_{\text {out }}, r_{t r}, \alpha, \beta$ and $\delta$, the mass accretion rate $\dot{m}$, and neglecting pair production, the calculation proceeds as follows. We begin with a trial value for the advection parameter $f_{a v}$, and obtain the global dynamical solution. This gives the following gas properties in the various radial shells of the ADAF and the corona: $v_{j}, \Omega_{j}, c_{s, j}^{2}, \rho_{j}, n_{e, j}, Q_{j}^{+}$. Next we assign reasonable values to the electron temperature $T_{e, j}$ in all radial shells, and calculate the ion temperature $T_{i, j}$ via equation (2-6), and also the quantities $Q_{j}^{i e}$ and $Q_{j}^{e, a d v}$. We now compute the spectrum of radiation emitted by each radial shell via synchrotron, bremsstrahlung and Compton scattering. This calculation is done by the iterative scattering method of Poutanen \& Svensson (1996) as described in NBM. It includes full coupling between the ADAF and the thin disk, namely irradiation of the ADAF by disk radiation, irradiation of the disk by the ADAF, reflection from the disk, generation of the iron $\mathrm{K} \alpha$ line, etc. Once the radiation transfer problem is solved, we obtain $Q_{j}^{-}$corresponding to each shell and check whether the electron energy balance equation (2-7) is satisfied. If necessary, we iteratively modify the electron temperature profile $T_{e, j}$ until equation (2-7) is satisfied. Once this is achieved, we compute $f_{a v}$ via equation (2-2) and check if it agrees with our trial value. If not, we choose a new value for $f_{a v}$ and repeat the rest of the calculation described above.

To include pair processes we need to compute one additional quantity as a function of radius, namely the pair fraction $z_{j}$. This is done by solving equation (2-12) in each shell. However, this is not trivial and requires an additional iteration loop in the calculation just described because the radiation solution is sensitive to $z_{j}$ and the pair production rate, $\left(\dot{n}_{+}\right)_{\operatorname{prod}, j}$, depends on the radiation field.

In some cases, for instance when we want to determine the critical accretion rate $\dot{m}_{\text {crit }}$ (see $\S 3.3)$ or the coronal accretion rate $\dot{m}_{c, 0}$ (see $\left.\S \S 3.4,3.5\right)$, we are interested in a solution with a given value of $f_{a v}$. The calculation then proceeds as described above, except that $\dot{m}$ or $\dot{m}_{c, 0}$ is the adjustable parameter rather than $f_{a v}$. We start with a guess for $\dot{m}$ or $\dot{m}_{c, 0}$ and adjust it at each iteration to ensure that $f_{a v}$ computed via equation (2-2) is equal to the desired value.

The calculations presented here are a significant improvement over earlier versions. In particular, by introducing the average advection parameter $f_{a v}$, the dynamical solution is made consistent with the radiative transfer and cooling part of the calculation. The only serious approximation is that we set $f(r)$ equal to a constant, $f_{a v}$, whereas $f$ is in general expected to vary with radius (Esin 1997). In order to compute a realistic $f(r)$, we would need to include a radial energy transport term in the electron energy equation (2-7). Potential transport mechanisms include convection (cf. Narayan \& Yi 1994, 1995a; 
Igumenshchev, Chen, \& Abramowicz 1996) and ion and electron conduction. These transport phenomena can cause a significant flow of energy from one radius to another, and they are not easy to calculate from first principles. Furthermore, radial energy transport may tend to smooth out variations in $f(r)$, driving the system toward a constant $f_{a v}$. We therefore feel that it is reasonable to set $f(r)=f_{a v}$ in determining the global dynamics of the flow. In effect, we treat the ADAF under a one-zone approximation for the purposes of the advection parameter $f$, while calculating the detailed variation with radius of all other gas properties.

Note that our models satisfy two separate energy equations: an energy equation for the electrons given in equation (2-7), and an energy equation for the total gas (ions plus electrons) which is satisfied by the global flow solution (see Narayan et al. 1997; Chen et al. 1997). Thus, we treat both the ions and the electrons self-consistently. Nakamura et al. (1997) describe another approach where they write down individual energy equations for the ions and the electrons. The two approaches are in principle equivalent.

\section{Modeling the Spectral States of Black Hole X-Ray Binaries}

In this section we investigate the spectral properties of the model described in the previous section and demonstrate how the various spectral states observed in BHXBs can be understood in the context of this model. The application of the model to the quiescent state of BHXBs has been extensively discussed by NMY, NBM and Hameury et al. (1997). Here we summarize the main results of that work and use the quiescent state as the starting point to explore the effect of increasing the mass accretion rate. A preliminary discussion of some of the new results presented here may be found in Narayan (1996).

Figure 1 summarizes the broad features of the scenario we develop in detail in the succeeding subsections. The figure shows how the geometry of the flow changes as the mass accretion rate is varied, and identifies various well-defined stages in the evolution of the flow with the known spectral states of BHXBs. At the lowest $\dot{m}\left(\lesssim 10^{-2}\right)$ we have the quiescent state, where the thin accretion disk is truncated at a fairly large transition radius and the accretion flow switches to an ADAF farther in (the bottom panel in Figure 1). The physics which determines the exact value of the transition radius is not well understood, but it is likely to be related to the angular momentum of the material flowing in from the companion star. In low-mass BHXBs, where mass is transferred via Roche lobe overflow, the circularization radius of the incoming stream is large $\left(r_{\text {circ }} \sim 10^{4}-10^{5}\right)$. Making the reasonable assumption that $r_{t r}$ is smaller than $r_{\text {circ }}$ by a factor of a few, we expect in these systems $r_{t r} \sim 10^{4}$. In high mass BHXBs, on the other hand, the angular momentum 
of the accreting material is much lower since mass transfer is driven by winds and the gravitationally captured gas circularizes at a much smaller radius (cf. Frank et al. 1992). Thus, $r_{t r}$ may be as small as $10^{2}$ or even smaller in these systems.

The other panels in Figure 1 show the effect of increasing $\dot{m}$ above its quiescent range. For $\dot{m}$ up to a critical value $\dot{m}_{\text {crit }} \sim 0.08$ (the exact value depends on the value of $\alpha$ and $\beta, \S 3.2)$, the geometry remains essentially the same as in the quiescent state. However, since the radiative efficiency of the flow increases rapidly with increasing $\dot{m}$ (Narayan \& Yi 1995b), the flow becomes quite luminous. We identify such flows with the low state. Once $\dot{m}$ exceeds $\dot{m}_{\text {crit }}$, the hot ADAF zone radiates too efficiently to remain advection-dominated. As a result, the ADAF begins to shrink in size and the inner edge of the thin disk moves inward to smaller radii. We identify such flows, where the central ADAF is still present but with a reduced size compared to the quiescent and low state, with the intermediate state. At still higher $\dot{m}$, the central ADAF zone disappears altogether and the thin disk moves in all the way to the marginally stable orbit. A somewhat weak corona is present above the disk. We associate this configuration with the high state. Finally, at accretion rates close to Eddington we assume that the flow makes a transition to a different state where the corona is much more massive and active. We tentatively identify this flow configuration with the very high state, although this is the weakest aspect of our scenario.

In the calculations presented below, unless otherwise stated, we use the "standard" parameter set summarized in Table 1. For those quantities that can be derived from observations we adopt system parameters corresponding to the SXT Nova Muscae 1991 (see $\S 4.1$ ). For the parameter $\delta$, we invariably choose a value of $10^{-3}$, but this quantity plays no role in the calculations presented here and could equally well be set to zero. This still leaves two important parameters, $\alpha$ and $\beta$. We choose what we consider to be the most natural values for these. We assume that the magnetic field is in equipartition with the gas pressure, which corresponds to $\beta=0.5$. The assumption of equipartition fields is very common in many areas of high energy astrophysics. In particular, equipartition fields are quite plausible in accretion flows since the Balbus-Hawley (1991) instability, which presumably is the mechanism whereby the field grows, is known to shut off when $\beta \sim 0.5$. For the viscosity parameter $\alpha$ we follow the prescription suggested by Hawley \& Balbus (1996, see also Hawley, Gammie \& Balbus 1995, 1996), viz. $\omega_{R \phi} \sim 0.5-0.6 p_{\text {mag }}$, where $\omega_{R \phi}$ is the shear stress. For $\beta=0.5$ this gives a value of $\alpha$ in the range $0.2-0.3$. We choose a value in the middle of the range, $\alpha=0.25$.

It should be emphasized that we have no adjustable parameters in the calculations presented in this paper except for the mass accretion rate $\dot{m}$ (and to a very limited extent $r_{t r}$ ), We could, in principle, optimize $\alpha$ and $\beta$ so as to obtain the best fit between the model 
and the Nova Muscae data discussed in $\S 4$, but we feel that the data are not really good enough for such an exercise.

\subsection{Quiescent State}

Between successive outbursts, transient BHXBs are generally found in the quiescent state, where the observed luminosity is many orders of magnitude below Eddington. In the systems for which optical and X-ray observations in quiescence exist (A0620-00, V404 Cyg, and GRO J1655-40), the data are explained quite well with the model shown in the bottom panel of Figure 1 (NMY; NBM; Hameury et al. 1997). In addition, the same model also explains observations of the underluminous supermassive black hole at the center of our Galaxy, Sgr A* (Narayan et al. 1995), as well as the supermassive black hole in NGC 4258 (Lasota et al. 1996a). These applications represent the most important successes so far of the ADAF model.

On the basis of the above work, we define the quiescent state of BHXBs to correspond to mass accretion rates $\dot{m} \lesssim 10^{-2}$. In Figure 2 we plot a sequence of spectra computed with our standard parameter set. The blackbody-like optical/UV peak is produced by self-absorbed synchrotron emission, while the peak at high energies $\sim 100 \mathrm{keV}$, visible especially clearly at very low $\dot{m}$, is due to bremsstrahlung. Inverse Compton scattering of synchrotron photons by the hot ADAF electrons is responsible for the rest of the spectrum and produces one or more bumps between the synchrotron and bremsstrahlung peaks.

With increasing $\dot{m}$, two effects modify the shape of the spectrum. Since gas pressure increases roughly linearly with $\dot{m}$, the magnetic field grows as $\dot{m}^{1 / 2}$ (equipartition); consequently, the synchrotron peak moves towards higher frequencies. At the same time, the photon spectral index in the $1-10 \mathrm{keV}$ X-ray band steepens from $\alpha_{N} \sim 1.7$ to $\sim 2.2$. This is because at low $\dot{m}$ high energy photons are produced primarily by bremsstrahlung emission, whereas at higher $\dot{m}$ Comptonization dominates, which results in a smoother but steeper spectrum. Since the radiative efficiency of the flow, defined as the ratio $\epsilon_{-1}=L_{b o l} / 0.1 \dot{M} c^{2}$, is proportional to $\dot{m}$ (Narayan \& Yi 1995b), the overall normalization of the spectrum changes roughly as $\dot{m}^{2}$. Even for $\dot{m} \sim 0.01$, the efficiency is quite low $\left(\epsilon_{-1} \sim 0.02\right)$ and these quiescent state models are very underluminous.

In the models shown here, the radiation from the outer thin disk is negligibly small compared to the emission from the ADAF. This would not be true if the transition radius were closer to the black hole, as we discuss at the end of $§ 3.2$ (see also Figure 5). 


\subsection{Low State}

The five heavy curves in Figure 3a show a sequence of model spectra where the flow geometry is exactly the same as in the quiescent state; now, however, $\dot{m} \geq 10^{-2}$ (Figure 1). At these relatively high accretion rates, Comptonization of synchrotron photons by the hot gas in the ADAF constitutes the dominant cooling mechanism. As $\dot{m}$ increases, the optical depth of the ADAF goes up causing a corresponding increase in the Compton $y$-parameter. Consequently, the high energy part of the spectrum becomes harder and smoother, and the photon index in the X-ray band reverts from $\alpha_{N} \sim 2.2$ back to $\sim 1.5$. The radiative efficiency for the highest $\dot{m}$ model is reasonably high and the total energy output is on the order of $10^{37} \mathrm{erg} \mathrm{s}^{-1}$, which corresponds to a few percent of the Eddington luminosity (for the assumed $6 M_{\odot}$ black hole). Most of the flux is emitted at around $100 \mathrm{keV}$ and the spectrum falls off exponentially at higher energies. The model spectra shown in Figure $3 a$ reproduce well both the spectral shapes and X-ray luminosities of BHXBs observed in the low state (Tanaka \& Lewin 1995; Tanaka \& Shibazaki 1996). Based on this correspondence, we identify these models with mass accretion rates $10^{-2} \lesssim \dot{m} \lesssim 10^{-1.1}$ with the low state.

The electron temperature profiles corresponding to our low state models are shown in Figure 3b. There is a clear anti-correlation between the mass accretion rate and the electron temperature in the inner part of the flow $\left(r \leq 10^{2}\right)$. This trend arises because, as $\dot{m}$ increases, a larger fraction of the dissipated energy is radiated away. The flow thus becomes more cooling dominated and its temperature decreases. We note that OSSE observations of the X-ray nova GRO J0422+32 in the low state showed an inverse dependence between the high energy cutoff energy and the $100 \mathrm{keV}$ flux (Kurfess 1996), as our low state models predict.

The decrease of $T_{e}$ with increasing mass accretion rate is important in considering pair production in the ADAF. At low $\dot{m}$ the radiative efficiency of the flow is small, and at high $\dot{m}$ the electron temperature is considerably below the pair creation threshold of $511 \mathrm{keV}$. Consequently, only the particle-particle pair production processes are important, which are very inefficient for $T_{e} \lesssim 10^{1} 1 K$. We obtain the equilibrium pair density of $z \sim 5 \times 10^{-7}$ at $\dot{m}=10^{-2}$ and $z \sim 6 \times 10^{-9}$ at $\dot{m}=\dot{m}_{\text {crit }}$. Our values of $z$ are significantly lower than those obtained by Kusunose \& Mineshige (1996) for ADAFs. The difference is most likely due to the fact that they considered only cooling by bremsstrahlung and inverse Compton scattering, and consequently obtained much higher electron temperatures.

Most of the emission in the low state is from the ADAF, and the outer thin disk is almost invisible. When $\dot{m}$ is low, the optical/UV peak in the spectrum is mostly due to self-absorbed synchrotron emission and the thin disk contribution is hardly seen. However, when $\dot{m}$ is high, the thin disk blackbody component becomes more prominent and is visible 
as a peak in the optical. The transition is especially clear in the spectrum corresponding to $\dot{m}=10^{-1.4}$, where both the synchrotron peak and the shoulder corresponding to the disk component are evident. This discussion pertains to models with a large transition radius, $r_{t r} \sim 10^{4}$. We discuss later the effect of a smaller transition radius.

One of the new effects we have introduced in the present model is the electron advection term in the energy balance equation (see $\S 2.2$ and Appendix A and the original discussion of Nakamura et al. 1997). To illustrate the effect of this term, we show by the thin lines in Figure 3a spectra computed without including electron advection. For high $\dot{m}$ the difference between spectra with and without electron advection is very small. However, the advective heating term depends linearly on $\dot{m}$, while the ion-electron Coulomb exchange rate, $Q^{i e}$, is proportional to $\dot{m}^{2}$. Thus, with decreasing $\dot{m}$ we expect that advection will become the dominant heating mechanism for the electrons. This trend is evident in Figure 3a. For $\dot{m}=10^{-2}$, for example, the inclusion of electron advection makes the system nearly twice as luminous as when the term is not included.

Figure 3b makes a similar comparison of the electron temperature profiles. The solid thin line shows $T_{e}(r)$ for $\dot{m}=10^{-2}$ when electron advection is not included. This is to be compared with the solid thick line which corresponds to the same model with electron advection included. We see that the addition of the electron advection term increases the electron temperature in the inner parts of the flow by acting as an extra heating term. In the outer parts of the flow, however, the electron temperature is reduced compared to its old value. Thus the energy output is increased in the inner parts of the flow, and decreased in the outer parts, and the emission becomes more centrally concentrated.

In Figures $4 \mathrm{a}$ and $4 \mathrm{~b}$ we demonstrate how low state spectra vary as a function of other model parameters. The effect of varying the inclination angle $i$ is illustrated in Figure $4 \mathrm{a}$ for $\dot{m}=10^{-1.4}$. When the system is viewed almost face-on $\left(i=5^{\circ}\right)$ it appears brighter by a factor of $\sim 2$ compared to an edge-on system $\left(i=90^{\circ}\right)$. Both the disk and the ADAF contribute to this effect. As $i$ increases, the projected area of the thin disk visible to the observer decreases as $\cos i$, and the corresponding blackbody component in the spectrum decreases proportionately. The hot ADAF is not completely spherical either, as the flow is flattened at the poles. This leads to an anisotropy in the optical depth from the center to the edge of the ADAF. Thus, the hot Comptonized photons from the center of the ADAF tend to escape preferentially along the rotation axis rather than along the equator, making face-on systems brighter. Clearly, this effect is important only for relatively large $\dot{m}$ when most photons are scattered at least once before escaping. At lower $\dot{m}$, the ADAF is optically thin and its emission is virtually independent of $i$ (except for relativistic Doppler factors, see Jaroszyński \& Kurpiewski 1997), and only the rather weak thin disk component 
varies with inclination.

The shape of the iron line is again a function of $i$. In our model the line width is simply proportional to $\sin i$, so that when the system is viewed face-on the $6.4 \mathrm{keV}$ feature is narrower and more prominent. In our model the equivalent width of the iron line has the same value (a few $\mathrm{eV}$ ) for all $i$, since we do not take into account the dependence on the viewing angle. In a more realistic calculation, the equivalent width falls off sharply for $i \gtrsim 60^{\circ}$ (George \& Fabian 1991).

In Figure $4 \mathrm{~b}$ we show how low state spectra depend on the values of $\alpha$ and $\beta$. The three spectra shown here each correspond to the critical accretion rate, $\dot{m}_{\text {crit }}$, the maximum $\dot{m}$ for which the low state is possible (see $\S 3.3$ for a more detailed discussion of $\dot{m}_{\text {crit }}$ ). The solid and dotted curves correspond to $\alpha=0.2$ and 0.3 , respectively. We see that an increase in $\alpha$ leads to an increase in the value of $\dot{m}_{\text {crit }}$ (the scaling is proportional to $\alpha^{2}$, see Narayan \& Yi 1995b), which increases the maximum luminosity that one can observe in the low state. In addition, the larger $\alpha$ also causes the spectrum to be harder. The dashed line in Figure $4 \mathrm{~b}$ demonstrates the effect of increasing $\beta$ from our standard value of 0.5 to 0.95 . Since $(1-\beta)$ is the ratio of the magnetic pressure to the total pressure in the ADAF, the change in $\beta$ causes the magnitude of the $B$-field to be reduced by a factor of $\sqrt{10}$. Consequently, the ADAF produces less synchrotron emission and this causes the electron temperature to go up in order to maintain the cooling rate. The increase in $T_{e}$ is clearly seen in Figure 4b: the spectrum corresponding to $\beta=0.95$ (indicated by the dashed line) is harder and cuts off at a higher energy than the equivalent $\beta=0.5$ model (dotted line). The spectrum also has a slightly harder slope.

In those BHXBs where the donor star is more massive than the accreting black hole (e.g. Cyg X-1) the mass transfer is often driven by a stellar wind from the companion. In this case the net angular momentum of the accreting material is low, and the circularization radius and the transition radius are likely to be much smaller than in low mass BHXBs (cf. Frank et al. 1992). In such systems, the emission from the thin disk in the quiescent and low states will be significantly enhanced. To illustrate this effect we show in Figure 5 a sequence of quiescent and low state spectra computed for a model with $r_{t r}=10^{2}$. The disk blackbody emission now clearly dominates over the synchrotron peak. Since the maximum disk temperature varies as $\propto r_{t r}^{-3 / 4}$ (Frank et al. 1992), the blackbody peak component is shifted into the far UV band for low $\dot{m}$ models, and into the soft X-ray band for higher $\dot{m}$. The appearance of the iron $\mathrm{K} \alpha$ line also changes with decreasing $r_{t r}$. Its width is proportional to $r^{-1 / 2}$ and its equivalent width depends sensitively on the solid angle subtended by the thin disk as viewed from the hot flow (see Figure 8 and the discussion in $\S 3.3)$. As a result, in wind-fed systems we expect both quantities to be significantly larger 
in the quiescent and low states compared to Roche lobe overflow systems.

\subsection{Low State to High State Transition: Intermediate State}

The hot two-temperature advection-dominated solution on which our models are based is known to exist only for accretion rates below a critical value $\dot{m}_{\text {crit }}$. For $\dot{m}>\dot{m}_{\text {crit }}$ the ratio of the cooling rate to the heating rate becomes so large that no hot equilibrium solution is possible. The topology of the solution space in the vicinity of $\dot{m} \sim \dot{m}_{\text {crit }}$ was described via a local analysis by Narayan \& Yi (1995b) and Chen et al. (1995).

According to the local analysis, for $\dot{m}<\dot{m}_{\text {crit }}$, there are actually two hot solutions (Narayan \& Yi 1995b). One of these is the ADAF solution which the present paper is based on, while the other is a hot two-temperature solution discovered originally by SLE. The SLE solution is a cooling-dominated solution which is thermally unstable (Piran 1978, Wandel \& Liang 1991). In terms of $f$, the ratio of advected energy to heat input, the ADAF solution corresponds to $f \rightarrow 1$ (for $\dot{m} \ll \dot{m}_{\text {crit }}$ ) while the SLE solution corresponds to $f \rightarrow 0$. As $\dot{m}$ approaches $\dot{m}_{\text {crit }}$ from below, the ADAF solution begins to radiate more efficiently and its value of $f$ decreases. At the same time, the SLE solution becomes more advection-dominated and its value of $f$ increases. At $\dot{m}=\dot{m}_{\text {crit }}$, the two solutions merge at an intermediate value of $f \sim 0.3$ and disappear. For $\dot{m}>\dot{m}_{\text {crit }}$ neither of the two hot solutions is present, and the only solution available is the cool thin accretion disk.

We find that the global solutions described in this paper have an exactly analogous behavior, except that the global advection parameter $f_{a v}$ which we defined in $§ 2.2$ replaces the local parameter $f$ of the Narayan \& Yi (1995b) analysis. To explore the topology of the solutions, we fixed $f_{a v}$ at various values between 0 and 1 and solved for $\dot{m}$ by the methods described in $\S 2.5$. Figure 6 a shows the result, and reveals that $\dot{m}$ is not a monotonic function of $f_{a v}$, but reaches a maximum value, which we call $\dot{m}_{\text {crit }}$, at $f_{a v} \simeq 0.35$. For $\dot{m}<\dot{m}_{\text {crit }}$, there are two solutions for $f_{a v}$. The solution branch with $f_{a v}>0.35$ corresponds to our global ADAF, while the branch with $f_{a v}<0.35$ represents the global

equivalent of the SLE solution. In analogy with the local analysis, we believe that only the ADAF branch represents a viable flow. The local instability of the SLE solution, which is known to be quite violent (Piran 1978), will likely carry over to the global SLE branch (though technically this has not been demonstrated), making this solution uninteresting for applications to real flows.

Figure 6 a shows that no hot solution is possible for $\dot{m}>\dot{m}_{\text {crit }}$. The exact value of $\dot{m}_{\text {crit }}$ depends in general on model parameters, e.g. it is proportional to $\alpha^{2}$ (Narayan \& Yi 
1995b, Abramowicz et al. 1995). However, we find that we almost always have $f_{a v} \sim 0.35$ at the critical solution. Therefore, a quick and convenient way of obtaining $\dot{m}_{\text {crit }}$ is to set $f_{a v}$ equal to 0.35 and to solve for $\dot{m}$. This is the procedure we have used in the calculations described below.

What happens when $\dot{m}>\dot{m}_{c r i t}$ ? A qualitative answer to this question is given by Esin (1997) who shows that in response to an increase in $\dot{m}$ the outer regions of the ADAF zone switch from the hot configuration to a thin disk, leaving the inner regions still in the hot configuration. In other words, the transition radius between the thin disk and the ADAF zone moves in and adjusts self-consistently to maintain an inner ADAF zone. In the context of the present global solutions, we can study this process by calculating $\dot{m}_{c r i t}$ as a function of the transition radius. The thick solid line in Figure $6 \mathrm{~b}$ shows the result, where each model was computed by fixing $r_{t r}$ to the desired value, setting $f_{a v}=0.35$, and determining the value of $\dot{m}_{\text {crit }}$ corresponding to the particular $r_{t r}$.

Consider now a flow that initially has $\log \left(r_{t r}\right)=3.9$ (our standard value, see Table 1) and whose $\dot{m}$ increases above $\dot{m}_{c r i t}\left(r_{t r}\right) \sim 0.083$ for this $r_{t r}$. The outer regions of the ADAF zone will cool and $r_{t r}$ will decrease. However, Figure $6 \mathrm{~b}$ shows that, as $r_{t r}$ decreases, the local value of $\dot{m}_{\text {crit }}$ becomes even smaller than it was at $r_{t r}=10^{3.9}$. Therefore, the ADAF continues to cool and shrink and to convert its outer parts into a thin disk. During this stage, the system configuration follows the short-dashed line (with the arrow pointing to the left). This continues until $r_{t r}$ reaches a value of about $10^{1.5}$. At this point, $\dot{m}$ becomes equal to the local $\dot{m}_{\text {crit }}\left(r_{t r}\right)$, and a stable self-consistent solution is possible. With further increases in $\dot{m}$, the system changes its $r_{t r}$ continuously by tracking the $\dot{m}_{c r i t}\left(r_{t r}\right)$ curve until $\dot{m} \sim 0.092$, when $r_{t r}$ reduces again discontinuously along the short-dashed line. At this point, the inner ADAF zone shrinks and disappears altogether, and for all $\dot{m}>0.092$ the thin disk extends down to the marginally stable orbit.

Figure 7 shows a sequence of spectra corresponding to the transition described above. The curves are labeled by $r_{t r}$, and are computed for the values of $\dot{m}$ given by the short-dashed line in Figure $6 \mathrm{~b}$. As $r_{t r}$ changes from $10^{3.9}$ to about $10^{1.5}$, the hard end of the spectrum is hardly affected because this radiation is produced by the inner regions of the ADAF which are insensitive to what is happening at larger radii. The soft blackbody peak due to the outer thin disk does change by a large amount and increases substantially in luminosity. However, this radiation is in the inaccessible EUV band. Therefore, based on X-ray observations alone, a system with $r_{t r} \sim 10^{2}$ looks almost identical to one with $r_{t r} \sim 10^{4}$, and would be classified as being in the low state. As $r_{t r}$ decreases below $10^{1.5}$, however, the spectrum changes more dramatically. Now, the cooling effect of the radiation from the disk Compton-scattering off the hot gas in the ADAF becomes more important 
and the ADAF emission becomes softer. At the same time the disk emission moves into the soft X-ray band and becomes an important component in the observed spectrum. Indeed, the radiation in the $\mathrm{X}$-ray band makes a remarkable switch from a very hard spectrum to a soft spectrum with an ultrasoft bump. The final model with $r_{t r}=10^{0.5}$ (indicated in Figure 7 by a dot-long-dashed line) is reminiscent of the high state observed in several BHXBs (e.g. Tanaka \& Shibazaki 1996). Therefore we identify the sequence of models discussed here with the famous low state to high state transition observed in several BHXRs. In our model, the transition is primarily due to a change in $r_{t r}$ (Figure 1), driven by a small change in $\dot{m}$.

Sudden changes from the low to the high state through a series of so called "intermediate state" spectra have been observed in several black hole systems, e.g. Cyg X-1 (Belloni et al. 1996), Nova Muscae (Ebisawa et al. 1994), and GX 339-4 (Mendez \& van der Klis 1997). Our model reproduces well several characteristics of the observations; for example, the bolometric luminosity does not change very much during the transition. There are two reasons for this. First, the change in $\dot{m}$ across the transition is quite small, $\sim 10 \%$. Second, the radiative efficiencies at the two ends are not very different. The low state, despite having an ADAF, has a fairly high radiative efficiency when $\dot{m}=\dot{m}_{\text {crit }}: \epsilon_{-1}=1-f_{a v} \sim 0.65$ (since $f_{a v}=0.35$ for the critical model). The high state model has an efficiency of $\sim 1$. Thus, the total change in the efficiency is only $\sim 30 \%$ and the change in the bolometric luminosity is no more than about 50\%. In fact, the observed change will be even less than this since the high state has most of its emission in an ultrasoft component, a part of which is not visible due to low-energy energy absorption by a detector window or the ISM. Therefore, if one considers say the $1-100 \mathrm{keV}$ band, the observed luminosity hardly changes at all during the transition. This feature has been noted by Zhang et al. (1997) in a low-high transition observed in Cyg X-1. Another very interesting feature of the model is that during the transition the spectrum pivots around a fixed luminosity at $10 \mathrm{keV}$ (see Figure 7). This again has been observed (e.g. E94, Zhang et al. 1997).

In addition to the broad features discussed above, other signatures in the spectra may be used to follow the change in $r_{t r}$ during the transition. X-ray reflection from the disk increases in magnitude as $r_{t r}$ decreases and this can be discerned in the shape of the hard X-ray continuum. Also, the strength and shape of the iron fluorescence line changes significantly. Figure 8 shows the variation of the line equivalent width. With decreasing $r_{t r}$, the fraction of the high energy photons incident on the thin disk increases from $\sim 1 \%$ to $30 \%$. On the other hand, when $r_{t r} \lesssim 10$ the incident spectrum steepens, so that there are fewer photons available above the iron K-edge. The net result is that the equivalent width of the line increases by roughly an order of magnitude as $r_{t r}$ moves in from $10^{3.9}$ to $10^{0.5}$. The line width, however, also increases as $r_{t r}^{-1 / 2}$ due to the Keplerian rotation of the inner 
edge of the disk. Therefore, while the equivalent width increases, the line also becomes physically much broader.

So far we have discussed a system that makes a transition from low to high values of $\dot{m}$. In SXTs, we usually consider the opposite case. In outburst, these systems quickly reach a nearly Eddington level of accretion and then undergo a steady decrease of $\dot{m}$ during the decline phase of the outburst. When these systems approach and cross $\dot{m}_{\text {crit }}$, they make a high to low state transition, moving along the long-dashed lines with arrows pointing to the right in Figure $6 \mathrm{~b}$. Because the $\dot{m}_{\text {crit }}\left(r_{t r}\right)$ curve is not monotonic, this track is slightly different than in the previous case. The difference could in principle be observed as hysteresis in the light curves or spectra. Systems like Cyg X-1 and GX 339-4 (cf. Grebenev et al. 1991) which move back and forth through the high-low state transition may be good candidates to study in this connection.

The results in Figure 6b, however, depend somewhat sensitively on our assumptions. The fact that the high-low transition occurs over a narrow range of $\dot{m}$ is probably a robust result, as are the broad features of the spectra shown in Figure 7. However, the exact shape of the $\dot{m}_{c r i t}\left(r_{t r}\right)$ curve in Figure $6 \mathrm{~b}$ depends on details. For instance, the dotted line in this figure shows what happens if we assume that half the energy required to evaporate gas from the disk into the the corona comes from the energy budget of the thin disk; our standard assumption (represented by the solid line), on the other hand, is that all the evaporation energy is supplied by the hot flow (see $§ 2.2$ ). There is a fairly significant change in the curve. Furthermore, even if the shape of $\dot{m}_{c r i t}\left(r_{t r}\right)$ is known accurately, the exact behavior of the system during the transition will still depend on the various timescales such as the time scale on which $\dot{m}$ changes, the thermal time scale of the hot flow, and the evaporation time scale for the thin disk. In plotting the curves in Figure $6 \mathrm{~b}$ we have assumed that the latter two are much shorter than the first; however, detailed numerical simulations are required to treat these effects properly.

\subsection{High State}

For accretion rates higher than the values discussed in the previous subsection ( $\dot{m} \gtrsim 0.092$ ), a pure ADAF zone is not possible for any choice of $r_{t r}$. Therefore, the only flow configuration allowed is a thin accretion disk that extends down to $r=3$, with a hot corona above it (Figure 1). To compute spectra of such models, we need a prescription for the mass accretion rate in the corona. As usual we assume that the corona behaves like an ADAF and we take the functional dependence of $\dot{m}_{c}$ to be of the form given in equation (2-1) with $r_{t r}=3$ (the marginally stable orbit). This still leaves free the overall normalization 
constant $\dot{m}_{c, 0}$. Now, we expect $\dot{m}_{c, 0}$ to be determined primarily by a competition between two major processes: (1) the evaporation of matter from the thin disk tends to boost $\dot{m}_{c, 0}$, and (2) radiative cooling of the hot gas causes $\dot{m}_{c, 0}$ to decrease. In analogy with the discussion in the previous subsection, we feel that a reasonable prescription is that the corona has the maximum $\dot{m}$ that is allowed for the ADAF solution. Therefore, we adjust $\dot{m}_{c, 0}$ until the advection parameter $f_{a v}=0.35$ in the coronal ADAF. This provides a self-consistent prescription for the corona that does not involve any additional parameters.

Figure 9 shows the values of $\dot{m}_{c, 0}$ that we obtain as a function of the total mass accretion rate $\dot{m}$ using this prescription. We see that $\dot{m}_{c, 0}$ decreases quite steeply with increasing $\dot{m}$. This behavior is easy to understand. Comptonization of cool photons from the thin disk is the dominant cooling mechanism in the coronal gas. As $\dot{m}$ increases, the disk flux irradiating the corona increases. This causes the corona to cool more efficiently, and so the corona saturates at a smaller value of $\dot{m}_{c, 0}$.

A sequence of spectra corresponding to these disk plus corona models is shown in Figure 10, the curves being labeled by the total mass accretion rate $\dot{m}$. We see that the emission is completely dominated by the blackbody disk component, which peaks in the soft $\mathrm{X}$-ray band. In addition, there is a weak high energy tail which is produced by Compton scattering of disk photons in the hot corona. The temperature of the soft peak $(\sim 1 \mathrm{keV})$ as well as the overall normalization of the spectra (which correspond to X-ray luminosities of around $10 \%$ or more of Eddington) agree with observations of the high state in several black hole systems (e.g. Cyg X-1, Nova Muscae and GX 339-4). This is, however, not a new result since it has been generally accepted for many years that the high state corresponds to a standard thin accretion disk (e.g. Tanaka \& Shibazaki 1996).

One new result of our model for the high state is that we find the mass accretion rate in the corona decreases with increasing $\dot{m}$. As a result, we predict that the more luminous systems should have less high energy emission. This is is consistent with observations on Nova Muscae 1991 (E94, see $§ 4)$.

It should be mentioned that at the values of $\dot{m}$ considered here the inner regions of a standard thin accretion disk are subject to thermal and viscous instabilities (Frank et al. 1992). Since BHXBs are seen in the high state (e.g. Nova Muscae, see $\S 4$ ) it is clear that the instabilities do not pose a serious threat to real systems. How do the systems survive? It has been suggested that perhaps the viscous stress in a thin disk is proportional to $\alpha$ times the gas pressure, rather than $\alpha$ times the total pressure (e.g. Sakimoto \& Coroniti 1981). Such a prescription renders the disk stable to both the thermal and viscous instability. Magnetic fields do not alter the stability provided the magnetic pressure scales with the gas pressure, as we have assumed. Incidentally, radiation pressure is small in the ADAF zone of 
all the models described in this paper; our ADAFs are therefore stable (Kato, Abramowicz \& Chen 1996).

\subsection{Very High State}

Some BHXBs, e.g. Nova Muscae 1991 (E94) and GX 339-4 (Miyamoto et al. 1991), have been observed to reach luminosities close to the Eddington luminosity, which is higher then the luminosities typically seen in the high state. In this "very high state," the spectra are found to be significantly harder than in the high state. A substantial fraction of the flux is emitted in a hard X-ray tail extending to well over $100 \mathrm{keV}$, with $\alpha_{N} \sim 2.5$.

The model we have described so far cannot explain the very high state. As we showed in $\S 3.4$, with increasing $\dot{m}$ the coronal emission actually decreases in the high state so that by the time we approach the Eddington mass accretion rate there is practically no hard component in the spectrum. Clearly we need a new idea to explain the very high state.

One ad hoc idea which has been quite popular for models of active galactic nuclei is to invoke enhanced energy dissipation in the corona, as suggested by Haardt \& Maraschi (1991). A possible mechanism could be coronal reconnection of magnetic field loops that are buoyantly ejected from the thin disk (Field \& Rogers 1993). We have attempted to incorporate this possibility in our models by introducing a new parameter $\eta$, defined as the fraction of the disk viscous energy that is dissipated directly in the corona; the models described so far in the paper correspond to $\eta=0$. By setting $\eta>0$ we can increase the coronal emission in our model by a fairly large factor. Not only does more of the energy go into the corona, a larger $\eta$ also results in a larger fraction of the mass being accreted via the corona. This is because the disk now produces less soft photons and so the the hot gas in the corona undergoes less Compton cooling. The coronal density thus increases above the value obtained for $\eta=0$.

Figure 11 illustrates the effect of varying $\eta$. We show spectra corresponding to two values of $\dot{m}$, in each case for models with $\eta=0.1,0.3$ and 0.5 . When we increase $\eta$ we transfer energy from the thin disk into the corona, and we see that this results in an anti-correlation between the magnitude of the ultra-soft blackbody disk component and the hard X-ray emission between 10 and $100 \mathrm{keV}$. Note, however, that the cutoff energy for the hard tail is relatively low. These models are just not hot enough to produce significant emission at $100 \mathrm{keV}$, whereas observations indicate a substantial flux even at a few hundred keV (Gilfanov et al. 1993).

The modeling of the very high state is the most speculative part of this paper. The 
idea we have explored here, based on Haardt \& Maraschi (1991), is just one of a number of possible scenarios. One reason for the uncertainty is that the luminosities observed in the very high state are generally comparable to the Eddington luminosity (e.g., see the discussion of Nova Muscae in $\S 4$ ), so that radiation pressure is likely to be important. We do not include radiation pressure in our models. Moreover, if the gravitational energy of the thin disk is indeed dissipated in the corona via magnetic field reconnection, the resulting particle distribution could be significantly non-thermal, which would modify the shape of the emerging spectrum.

\section{Outburst of Nova Muscae 1991}

Having developed an understanding of the various spectral states in BHXBs, and the relationship of the states to one another, we now compare the predictions of the model against observations of the soft X-ray transient Nova Muscae 1991. We discuss the binary parameters of Nova Muscae in $\S 4.1$ and describe the spectral data in $\S 4.2$. We then use our models to generate synthetic light curves and compare them against the observations in $\S 4.3$. Finally, we compare theoretical and observed spectra in $\S 4.4$.

\subsection{Optical Observations in Quiescence: Model Parameters}

After Nova Muscae returned to quiescence following its outburst in the fall of 1991, it was extensively studied by two optical observing teams and this work has provided a detailed and confirmed picture of the system. The binary has an orbital period of $\mathrm{P}=$ 10.4 hours and a mass function of $\mathrm{f}(\mathrm{M})=3.01 \pm 0.15 \mathrm{M}_{\odot}$ (Orosz et al. 1996; see also Casares et al. 1997). The mass ratio is $\mathrm{q}=\mathrm{M}_{2} / \mathrm{M}_{1}=0.133 \pm 0.019$ (Orosz et al. 1994), a result that has been confirmed using an independent technique (Casares et al. 1997). Two measurements of the inclination angle are in agreement and give a value of $i \approx 60^{\circ}$ (Orosz et al. 1996; Shahbaz et al. 1997). The limits on $i$ given by Orosz et al. are $54^{\circ}<i<65^{\circ}$. The corresponding mass and mass limits for the primary are $M_{1}=6.0 \mathrm{M}_{\odot}$ and $5.0<M_{1}<7.5 M_{\odot}$ (Orosz et al. 1996; see also Casares et al. 1997).

We estimated the distance to Nova Muscae as follows. The apparent magnitude of the K3-K5 secondary corrected for reddening and for the light contribution of the accretion disk is $\mathrm{V}_{0} \approx 20.0$ (Orosz et al. 1996). Using the dynamical data summarized above, Eggleton's (1983) formula for the radius of the Roche lobe, and Kepler's Law we computed the mean radius of the Roche-lobe-filling secondary: $R_{2}=1.03 R_{\odot}$. Finally, using the radius and 
apparent magnitude of the secondary, and the visual absolute flux of a K4 dwarf (Popper 1980) we find $\mathrm{d}=5.7 \pm 1.0 \mathrm{kpc}$. About half the estimated uncertainty is due to the uncertainty in $M_{1}$ given above; the remainder is due to uncertainties in the spectral type of the secondary and in the fraction of the total light that is contributed by the accretion disk. A virtue of this method of determining the distance is that it makes no assumption whatsoever about the uncertain evolutionary state of the secondary star. A somewhat greater distance is obtained by this method if one adopts the best-fit values of the mass function, $\mathrm{f}(\mathrm{M})=3.35 \mathrm{M}_{\odot}$, and orbital inclination, $i=54^{\circ}$, obtained by Casares et al. (1997) and Shahbaz et al. (1997), respectively: d =6.2 kpc. On the other hand, Shahbaz et al. (1997) argue from evolutionary considerations that the secondary star must be less massive than a main-sequence K3-K5 star, from which they conclude that $\mathrm{d}<4.0 \mathrm{kpc}$. For the purposes of this study, we adopt $\mathrm{d}=5.0 \mathrm{kpc}$.

In summary, as inputs to our model calculations we adopt $i=60^{\circ}, M_{1}=6.0 \mathrm{M}_{\odot}$, and $\mathrm{d}=5.0 \mathrm{kpc}$. In addition, from a study of the $\mathrm{H}_{\alpha}$ emission line profile we adopt $\mathrm{v}_{\text {in }}=2000$

$\mathrm{km} \mathrm{s}^{-1}$, which implies $\mathrm{r}_{t r}=8400$, i.e. $\log r_{t r}=3.9$ (NMY; Orosz et al. 1994). We take the outer edge of the thin disk to be at $80 \%$ of the Roche lobe radius of the black hole, which gives $\log r_{\text {out }} \sim 4.9$.

\subsection{X-ray Observations in Outburst}

The outburst of Nova Muscae 1991 was first detected on January 8 by the Ginga ASM (Kitamoto et al. 1992). It was subsequently observed in detail by the ASM and by the Ginga LAC for about 8 months (E94). Here we give an abbreviated account of its spectral evolution based on the works just cited (see also Brandt et al. 1992; Lund 1993; Goldwurm et al. 1992; Gilfanov et al. 1993).

Figure 12a shows two lightcurves of Nova Muscae, one corresponding to a standard X-ray band (2-12 keV, solid circles) and the other to a soft $\gamma$-ray band (20-100 keV, open circles). These light curves were computed by straightforward integration of the 51 Ginga Lac spectra described below, augmented with SIGMA data where available. The following is a brief description of the lightcurves based on the detailed accounts given by Kitamoto et al. 1992, E94 and Figure 12a.

Seven days after its discovery, Nova Muscae reached its maximum intensity of 8 Crab (1-6 keV). During this rising phase, the spectrum was initially dominated by a hard power-law component with a photon index $\alpha_{N} \sim 2.5$; however, as the total X-ray flux increased, the hard flux ( $\gtrsim 8 \mathrm{keV}$ ) gradually decreased. From about the time of maximum 
and for about four months thereafter, the ultrasoft blackbody component was dominant. For about 80 days following the maximum, this soft component decayed exponentially with a characteristic time scale of 31.2 days. Meanwhile, the hard flux decayed by more than two orders of magnitude. However, at about 80 days after the outburst the hard component began to rise in intensity, and a second broad maximum was observed in the soft component, which approximately doubled in intensity. Thereafter the soft component decayed more rapidly with an e-folding time of 21.9 days (Kitamoto et al. 1992). About 60 days after the outburst, the hard power-law component almost disappeared completely for a month. Then at about 130 days the spectrum underwent a high-low state transition during which the blackbody component softened and the power-law tail brightened substantially and became much harder $\left(\alpha_{N} \sim 1.6\right)$ than at any previous time during the outburst. This same hard spectrum was observed during the remaining eight observations by the Ginga LAC, which spanned the period from 157 to 238 days after the outburst (E94).

Spectral fitting parameters characterizing 51 spectra, which were derived from Ginga LAC observations, are summarized in Table 2 of E94. Each set of parameters was obtained by fitting the $1.2-37 \mathrm{keV}$ Ginga LAC pulse-height data to a spectral model composed of a soft disk blackbody component plus a hard power-law component. Ebisawa et al. used a total of seven spectral parameters: the blackbody temperature and normalization, the power-law photon index and normalization, the column density $\mathrm{N}_{H}$, and two parameters describing a broad absorption feature above the Fe-K edge. Using the values of these spectral parameters given by Ebisawa et al., we computed six representative spectra of Nova Muscae over the interval 1-40 keV. Specifically, we used the four blackbody and power-law parameters mentioned above. We set $\mathrm{N}_{\mathrm{H}}=0$ to approximate the intrinsic source spectrum, and we ignored the Fe-K absorption feature.

These six best-fit Ginga Lac spectra are shown in Figure 14 as heavy solid lines extending from 1-40 keV (1-10 keV for Figure 14b). The pairs of lighter solid lines, which flank each heavy line, give upper and lower limits on the spectra. The upper (lower) limit was obtained by varying all four spectral parameters simultaneously by $1 \sigma$ in order to maximize (minimize) the sum of the blackbody and power-law fluxes at all energies. These limits should be conservative, except possibly in the case of the spectrum for day 79 for which the fit to the data is unsatisfactory (see Table 2 in E94). The dotted extension of the heavy solid line in Figure 14a and Figures 14c-f is an extrapolation of the Ginga spectrum to $300 \mathrm{keV}$. The rationale for this extrapolation is the good agreement during the first 30 days of the outburst between the photon index determined by Ginga (E94) and the index determined by SIGMA (Goldwurm et al. 1992). In Figure 14b, the heavy line extends only from 1-10 keV because Ebisawa et al. were unable to determine the photon index of the hard component during this period and they fixed the photon index at 2.6. In the 10-40 
$\mathrm{keV}$ range, this spectrum is represented in Figure 14b by a dotted line which does not extend to higher energy. The triple of UV data points in Figures 14a-c are interpolated in time from the compilation of fluxes given by Shrader and Gonzalez-Riestra (1993).

\subsection{Modeling the X-ray and $\gamma$-ray Lightcurves}

As shown in Figure 12 and described in $§ 4.2$, and in E94, the bolometric luminosity of Nova Muscae reached a maximum around day 7 and then declined over the next several months. The decay of the $2-12 \mathrm{keV}$ X-ray flux was relatively smooth and monotonic, whereas the 20-100 keV $\gamma$-ray flux went through quite large variations. This behavior is readily interpreted in terms of the sequence of spectral states described in $\S 3$. Very High State: Before day $\sim 60$, the X-ray and $\gamma$-ray fluxes were intense; $\dot{m}$ was near or even somewhat greater than the Eddington rate for our adopted distance of $5 \mathrm{kpc}$. High State: From day 70 to day 120, the $\gamma$-ray flux was extremely faint while the X-ray flux remained fairly intense. Intermediate State: Around day 130 the source spectrum switched dramatically from soft to hard and the $\gamma$-ray flux became much larger than the soft flux. This was clearly a high-low state transition. Following this, from day 130 to day 200, both the X-ray and $\gamma$-ray fluxes remained fairly constant. Low State: From day 200 onward, the source maintained a very hard spectrum. On day 238, the last Ginga LAC observation, the source was quite faint relative to the peak of the outburst. By this time Nova Muscae was well into decline and approaching the quiescent state.

Thus, there is a qualitative relationship between the spectral states described by our model (§3) and the behavior of the X-ray and $\gamma$-ray light curves. We now seek a quantitative relationship between the model and the lightcurve data by specifying a mapping between the models described in $\S 3$ and the time of observation. Ignoring the very high state, our models are specified by only two parameters: (1) the mass accretion rate $\dot{m}$, which determines which of the four states the system is in, and (2) the transition radius $r_{t r}$, which follows the track shown by the long-dashed line in Figure $6 \mathrm{~b}$. In fact, $r_{t r}$ is an independent parameter only in the intermediate state. In all other states it has a specific value, either $r_{t r}=3$ (high state), or $r_{t r}=10^{3.9}$ (low and quiescent state).

The data in Figure 12a indicate that the luminosity in the $2-12 \mathrm{keV}$ band, which dominates the X-ray emission in the first four months following the maximum, declines roughly exponentially with time (see $\S 4.2$ ). The theoretical work of Cannizzo (1993) also suggests that the mass accretion rate during decline following a dwarf nova instability will have an exponential dependence under plausible conditions. We therefore assume an exponential scaling of $\dot{m}$ with time. In the intermediate state, we expect that it takes a 
finite period of time for the transition radius to move out from $r_{t r}=3$ (corresponding to the high state) to $r_{t r}=10^{3.9}$ (low state). Unfortunately we have very little understanding of the time dependence of $r_{t r}$, because of large uncertainties in the mechanism responsible for disk evaporation. In analogy with the $\dot{m}$ scaling we make the rather ad hoc assumption that $r_{t r}$ also has an exponential time dependence. We then obtain the following very simple mapping between our model parameters and the time of observation:

$$
\text { Time }-7 \text { days }=-t_{\dot{m}} \ln \left(\frac{\dot{m}}{\dot{m}_{0}}\right)+t_{t r} \ln \left(\frac{r_{t r}}{3}\right) .
$$

This prescription involves three parameters: (1) The mass accretion rate at maximum luminosity (day 7 ), $\dot{m}_{0},(2)$ the e-folding decay time of the mass accretion rate, $t_{\dot{m}}$, and (3) the e-folding time of the transition radius, $t_{t r}$ (the last parameter is needed only in the intermediate state).

As explained in $\S 3$, we are confident that our model captures the essential physics of four of the five spectral states, viz. the high state, the intermediate state, the low state and the quiescent state, but we are less comfortable with our proposal for the very high state as it involves a somewhat ad hoc assumption about the disk corona. Therefore, we concentrate first on the portion of the lightcurves from day 70 (where we place the transition between the very high and high states) onward.

By inspection of the light curves it is straightforward to estimate the three parameters in equation (4-1). First, during the period from day 200 to day 238, the source was in the low state, which in our model is dominated by the ADAF. Since the model predicts how the luminosity of the ADAF varies as a function of $\dot{m}$, the ratio of the fluxes on day 200 and day 238 immediately gives the change in $\dot{m}$ between these two points. From this we estimate that $t_{\dot{m}} \sim 35$ days. Next, we see that the source made the high-low transition around day 130. In our model this happens when the mass accretion rate has a value $\dot{m} \sim 0.092$ (see Figure $6 \mathrm{~b}$ ). This fixes the normalization of the mass accretion rate: $\dot{m}_{0} \sim 3$. Around day 130 the source first entered the intermediate state when the thin accretion disk began to shrink back and the central region was replaced by an ADAF. With time, the ADAF grew. According to the model, the high-low transition occurs during the period when $r_{t r}$ increases from $10^{0.5}$ to $\sim 10^{1.5}$ (see Figure 7 ). Beyond this point, the X-ray spectrum should remain basically unchanged while the transition radius continues to grow to its maximum value $\left(r_{t r}=10^{3.9}\right.$ in our model $)$. Indeed, the data show such behavior; following the high-low transition on day 130, there is a pronounced plateau in both the X-ray and $\gamma$-ray lightcurves extending up to day 200. If we assume that the ADAF reached its maximum size on day 200, then we immediately obtain an estimate of the third parameter: $t_{t r} \sim 8$ days.

Now that the three mapping constants are known, the model is completely specified; 
Figure $12 \mathrm{~b}$ shows the variations of $\dot{m}$ and $r_{t r}$ with time for the particular constants derived above. We are now in a position to calculate theoretical light curves. For each model described in $§ \S 3.1-3.4$ we use eq (4-1) to calculate the corresponding time coordinate in the light curve. Then, from the calculated model spectra we compute the luminosities in the 2-12 keV and 20-100 keV bands. The heavy and thin solid lines in Figure 12a show the theoretical X-ray and $\gamma$-ray lightcurves that result from this calculation.

The overall agreement between the theoretical lightcurves and the data is quite good, and the model reproduces most of the key features in the data rather well. Recall that we used only the following three pieces of information to fit the mapping constants: (1) The start time of the intermediate state (day 130), (2) the end time of the intermediate state (day 200), and (3) the change in total flux between day 200 and day 238. Several other features of the lightcurves which were not fitted are successfully predicted by the model:

(1) Days 200-238: The model has the correct ratio of the X-ray to $\gamma$-ray flux in the low state. That is, the model predicts the correct spectral index on day 200 and also has the correct variation of spectral index between day 200 and day 238 (see also Figure 12d and the discussion of the spectral index time variation below).

(2) Days 130-200: The model predicts the correct flux in the intermediate plateau in both the X-ray and $\gamma$-ray lightcurves (see Figure $12 \mathrm{a}$ ). Thus, the prediction that the intermediate state occurs at $\dot{m} \sim 0.08$ seems to be verified by the observations. Actually, the predicted fluxes are somewhat high, but this could be easily corrected by a slight change in the assumed black hole mass or the distance to Nova Muscae, or by reducing $\alpha$ slightly (since flux $\left.\propto \dot{m}_{\text {crit }} \propto \alpha^{2}\right)$.

(3) Days 70-130: The model does a surprisingly good job in the high state. In particular, the very different slopes of the X-ray and $\gamma$-ray lightcurves are predicted correctly. The X-ray flux is nearly proportional to $\dot{m}$ (except for a modest bolometric correction) because the high state spectrum is dominated by the ultrasoft component. It is pleasing that although the slope of the X-ray lightcurve here was not used to derive any of the parameters (recall that the $\dot{m}$ decay time scale of 35 days was obtained by considering the data beyond day 200), nevertheless the model fits the observations quite well. However, the overall normalization of the X-ray flux is about a factor of two less than the observations. The agreement in the $\gamma$-ray lightcurve is more impressive. As explained in $\S 3.4$, the model predicts that the coronal emission in the high state should increase with decreasing $\dot{m}$ and this prediction is well confirmed by the data.

We now consider the first 70 days of the outburst when Nova Muscae was in the very high state. As described in $§ 3.5$, to reproduce the very high state we need to invoke an extra 
parameter, $\eta$, which is the fraction of the disk gravitational energy that is dissipated in the corona. Since the exact mechanism for such energy transfer is not understood, the value of $\eta$, as well as its dependence on $\dot{m}$, are not known. A conservative assumption is that $\eta=0$ below some value of $\dot{m}$ and changes to a constant non-zero value for higher accretion rates. The solid and dashed curves in Figure 12a correspond to two such models where we have set $\eta=0.3$ and 0.1 , respectively, and where we have assumed that the transition between the very high and high states occurred around day 70. (Thus there are two additional parameters in our model of the very high state: $\eta$ and the day of the transition.) Both models reproduce the observed $\gamma$-ray lightcurves during the first 70 days reasonably well. However, they predict an abrupt change in the $\gamma$-ray flux on day 70 (because of the abrupt change in $\eta$ ), whereas the data show a gradual transition.

Perhaps a more physical model, but one that involves too much freedom in fitting the data, is one that allows $\eta$ to vary with $\dot{m}$ in a smooth fashion. The dotted line in Figure $12 \mathrm{a}$ shows a theoretical lightcurve where we have adjusted $\eta$ to achieve a good agreement with the data during the entire period of the very high state except near day 10; note that we still set $\eta=0$ once the system goes into the high state. Figure 12c shows our three models for $\eta$ as a function of time. Clearly the variable $\eta$ model gives a better fit to most of the observed flux points in Figure 12a, but it is not clear if there is any physical significance to the derived run of $\eta(t)$.

As a further test of the models we show in Figure $12 \mathrm{~d}$ the predicted variation of the photon index of the power-law component of the spectrum (calculated between 10 and 20 $\mathrm{keV}$ ) compared with the photon indices measured by E94. The gap in the data between day 50 and day 130 corresponds to the time during which Nova Muscae was too faint at high energies for E94 to determine the photon index. Figure 12d shows that the model is in very good agreement with the measured indices in the intermediate and low states (days 130 to 238). In the very high state, the $\eta=0.3$ model gives a reasonable fit, while the $\eta=0.1$ model (not surprisingly) predicts a power-law tail that is too steep. Oddly enough, the variable $\eta$ model, which gives the best fit to the broad band fluxes, does not fit the observed photon indices very well.

Finally, Figure 13a shows the bolometric (solid line), UV (dashed line) and optical (dotted line) lightcurves of Nova Muscae during the outburst, computed from the model. Figure $13 \mathrm{~b}$ gives the radiative efficiency normalized to $10^{-1}$. All three curves in Figure 13a show a small step near day 70 which is associated with the transition from the very high state to the high state. In the very high state considerable energy is required to evaporate gas from the disk to maintain the massive corona; thus the radiative efficiency $\epsilon_{-1}$ of this state is reduced relative to the high state, as demonstrated in Figure 13b. Therefore, for a 
given value of $\dot{m}$, the very high state is somewhat less luminous than the high state. The X-ray flux in Nova Muscae did show a secondary maximum between days 70 and 80 (see Figure 12a), and indeed such features are common in many SXTs during decline (Tanaka \& Lewin 1995).

Another feature in the bolometric lightcurve in Figure 13a is the plateau corresponding to the intermediate state between day 120 and 200. This is the period during which $\dot{m}$ does not change very much but $r_{t r}$ changes by a large factor. As we explained in $\S 3.3$, the bolometric luminosity changes by only about $50 \%$ during this entire transition, which lasted about 80 days in the case of Nova Muscae. During the same period, there is a noticeable rise in the optical and UV flux from the system. This emission is produced in the outer parts of the thin disk. As the transition radius increases, the outer thin disk experiences more irradiation by the ADAF and this causes the disk temperature and UV/optical luminosity to increase considerably. A similar increase was seen in the optical lightcurve of A0620-00 during its 1975 outburst (see van Paradijs \& McClintock 1995 and references therein).

Finally, once the low state is reached, the bolometric luminosity drops very rapidly because by this point the flow is dominated by the ADAF. As Narayan \& Yi (1995b) showed, a characteristic feature of an ADAF is a steep decrease of radiative efficiency with decreasing $\dot{m}$ (Figure 13b), which causes the luminosity to fall rapidly. A sudden drop in the luminosity at late time was seen in Nova Muscae (Figure 12a), and also in other soft X-ray transients (Tanaka \& Shibazaki 1996 and references therein). By comparison, the optical and UV fluxes decrease much more slowly until about day 300. During this time the system is in the low state when the optical and UV emission is produced by self-absorbed synchrotron. As is clear from Figure 3a, the flux in this part of the spectrum decreases more slowly with $\dot{m}$ than the X-ray flux. However, after day 300 , the system is the quiescent state, where the total flux is dominated by the synchrotron emission. When, following the decline in $\dot{m}$, the synchrotron peak moves outside the UV band, the UV flux falls off more rapidly than the bolometric luminosity. The corresponding rapid decline occurs somewhat later in the optical light curve.

To summarize, we feel that our simple three-parameter $\left(\dot{m}_{0}, t_{\dot{m}}, t_{t r}\right)$ model reproduces the main features of the observations of Nova Muscae 1991 extremely well. By varying $\dot{m}$ and $r_{t r}$ in a systematic way we are able to follow the system through more than three orders of magnitude in the observed flux. There is only one minor problem, namely the predicted $\mathrm{X}$-ray flux is lower than the data in the high state. This could probably be fixed by using a better model for the thin disk, e.g. including the effects of electron scattering in the upper layers of the disk (Shimura \& Takahara 1995). 


\subsection{Modeling the Spectral Evolution of the System}

We computed model spectra that correspond in time with the six observed spectra discussed earlier ( $\S 4.2$; Figure 14). Our model spectra are shown as dashed lines in Figure 14. Note that there are no adjustable parameters here other than the three mapping constants which were fitted in the previous section. Note, in particular, that we have not normalized any of the predicted spectra. (If we allowed ourselves a normalization constant in each panel we would obviously get a better fit.)

The comparison shows that the model gives a good description of the spectral evolution of the system. This is not surprising, of course, since we have already shown that the model fits the light curves well. The purpose of this section is, in fact, not to demonstrate the goodness of fit but rather to use the spectral comparison to identify problem areas where further work is needed.

First, it is clear that the model seriously underpredicts the optical/UV flux (by a factor of 2 to 3 ) in the cases where data are available (panels $\mathrm{a}-\mathrm{c}$ ). This problem is most likely related to our simplified treatment of the thin disk. In computing the irradiation of the disk by the ADAF/corona, we assume that the disk is flat and infinitely thin (see NBM). However, a real disk is expected to be flared on the outside, so that the disk will intercept a larger fraction of the irradiating flux than we allow in the model. It is straightforward to include disk flaring and it is clear that this will lead to an increase in the predicted optical/UV flux (e.g. Vrtilek et al. 1990). Whether or not the problem will be completely eliminated remains to be seen.

The second problem is that the model spectrum in the very high state (panel a) cuts off at quite a low energy $\sim 50 \mathrm{keV}$. This is not consistent with observations which indicate that the power-law component of the spectrum extends well over $100 \mathrm{keV}$ (Gilfanov et al. 1993), a result that is also confirmed by observations of V404 Cyg, GRO J0422+32, and GS2000+25 in the very high state (Sunyaev et al. 1993). This problem convinces us that the model of the very high state presented here is probably not correct.

In the high state (panel b), there is an apparent discrepancy between the high energy slopes of the predicted and observed spectra. However, the slope shown by the dotted line in this panel was assigned arbitrarily by E94 since they were unable to measure it from their data. Therefore, it is not clear that the discrepancy is real. There is also a small problem with the normalization of the soft component in the intermediate state (panel c); the problem is in fact slightly worse at the end of the high state (days 100 to 125). In general, the predicted soft $\mathrm{X}$-ray flux in the high state is too low by a factor of $\sim 2$. This is the same problem which we mentioned already in $\S 4.3$ in connection with the X-ray lightcurve. We 
are unsure at this point whether or not this indicates a serious difficulty with the model.

The remaining panels in Figure 14 all show quite good agreement between the model and the data. In the low state spectra shown in panels $\mathrm{d}-\mathrm{f}$, the apparent soft components in the observed spectra are quite uncertain, and the agreement between the model and the data is in fact good. The calculated low state spectra cutoff at around $100 \mathrm{keV}$. The Nova Muscae data do not rule out such a cutoff (the dotted lines are just extrapolations of the Ginga data, since there were no observations at these energies available after day 30). In fact, observations of other SXTs in the low state seem to indicate an exponential cutoff near $100 \mathrm{keV}$, e.g. GRO J0422+32 (Kurfess 1996). We therefore believe that this feature of the model is correct.

\section{Discussion and Conclusions}

One of the major goals of high energy astrophysics is the development of a consistent predictive theory of accretion flows around black holes. Ideally, such a theory should start with a few basic parameters, such as the black hole mass $M$, its spin parameter $a$, the inclination of the system $i$, the outer radius of the accretion flow $r_{\text {out }}$, and the Eddington-scaled mass accretion rate $\dot{m}$, and predict the spectrum and variability of the system.

The thin accretion disk model of Shakura \& Sunyaev (1973) and Novikov \& Thorne (1973) (see Frank et al. 1992 for a modern review) was an important step in developing such a theory. Unfortunately, the model predicts a very soft blackbody-like spectrum with $k T \sim 2\left(M / M_{\odot}\right)^{-1 / 4} \dot{m}^{1 / 4} \mathrm{keV}$. While this spectrum fits observations of some accreting black holes, it does not explain many other systems that emit much of their radiation in hard $\mathrm{X}$-rays and $\gamma$-rays, with photon energies up to $100 \mathrm{keV}$ or more. Indeed, among the five spectral states that have been identified in BHXBs, namely the quiescent, low, intermediate,

high and very high states, the thin accretion disk model explains only the high state. The other four states all involve hot optically thin gas with electron temperatures $\gtrsim 10^{9} \mathrm{~K}$. Until recently there were no good dynamical models of accretion flows with these properties (see Liang 1997 for a review).

The situation has changed with the discovery of a new class of hot, optically thin, advection-dominated accretion flows (ADAFs) (Narayan \& Yi 1994, 1995b, Abramowicz et al. 1995, Chen et al. 1995), which have electron temperatures in the range needed to explain the observations, and which do not suffer from the strong thermal and viscous instabilities that plagued earlier attempts to develop hot accretion models (e.g. SLE, Piran 
1978, Wandel \& Liang 1991). ADAF models with low (Eddington-scaled) mass accretion rates, $\dot{m} \sim 10^{-3}-10^{-2}$, have been successfully used to explain the quiescent state of BHXBs (NMY, NBM, Lasota et al. 1996b, Hameury et al. 1997). The geometry of the flow is shown schematically in the lowest panel of Figure 1, and consists of an ADAF that extends from the black hole horizon to a transition radius $r_{t r} \sim 10^{4}$ (in Schwarzschild units) and a thin accretion disk that extends from $r_{t r}$ to an outer radius $r_{\text {out }}$. A similar model (either with or without the outer disk) also works for low luminosity galactic nuclei (Narayan et al. 1995; Fabian \& Rees 1995; Lasota et al. 1996a; Reynolds et al. 1996; Mahadevan 1997).

A preliminary study of the spectral states of BHXBs using the ADAF model was made by Narayan (1996). The present paper demonstrates that when the ADAF model of the quiescent state is extended to higher mass accretion rates it reproduces the low state, intermediate state, and high state of BHXBs and naturally explains the transition from the low state to the high state. Thus, the model presented here provides a unification of the quiescent, low, intermediate and high states and represents the first consistent and predictive theory that succeeds in encompassing a large fraction of the phenomenology of accreting black holes (but not all of the phenomenology since the very high state still remains to be explained).

The basic scenario that emerges from the present study is shown schematically in Figure 1 and is discussed in detail in $§ 3$. We propose that the low state is similar to the quiescent state in its flow geometry, but with a higher mass accretion rate. This state survives until $\dot{m}$ reaches a critical value which we calculate to be $\dot{m}_{c r i t} \sim 0.083$ (for values of $\alpha$ and $\beta$ given in Table 1). Once $\dot{m}$ crosses $\dot{m}_{\text {crit }}$, the ADAF zone begins to shrink and the thin disk moves in by a corresponding amount. By the time $\dot{m}$ reaches about 0.092 the disk moves all the way down to the marginally stable orbit and there is no central ADAF. An ADAF still persists, however, in the form of a corona above the disk. We identify the transition states, which correspond to mass accretion rates in the range $0.083 \lesssim \dot{m} \lesssim 0.092$, with the intermediate state. However, until the transition radius decreases to $r_{t r} \sim 10^{1.5}$, the X-ray spectrum of the system in the intermediate state is roughly the same as in the low state (see Figure 7); the spectrum begins to soften only for $r_{t r}<10^{1.5}$. Because of this, our theoretical definition of the intermediate state is somewhat different from the one adopted by the observational community; we assume that the system is in the intermediate state whenever $r_{t r}$ is smaller than its quiescent value, whether or not the X-ray spectrum softens. Finally, accretion flows with $\dot{m}>0.09$ correspond to the high state. The X-ray luminosities and spectra calculated with the above scenario are in good agreement with observations of BHXBs.

An important point which should be emphasized is that the scenario shown in Figure 
1 is an inevitable consequence of the ADAF model and is not put in by hand or adjusted in any way. This is because of two major features that set this model apart from previous studies of accreting black holes. First, the present model achieves a fairly high level of self-consistency in its combined treatment of dynamics and radiation processes $(\S 2.5)$. Specifically, the model (1) satisfies mass and momentum conservation at each radius by making use of global flow solutions (§2.2, and Narayan et al. 1997c, Chen et al. 1997), (2) solves for the electron and ion temperature at each radius of the accreting two-temperature gas by solving the energy equation $(\S \S 2.2,2.3)$, and (3) incorporates a fairly sophisticated treatment of radiative processes $(\S 2.4$, and NBM). Some loose ends do remain and there is need for further improvement as we discuss below. Nevertheless, we emphasize that a very high level of self-consistency has been achieved here. One consequence of the self-consistency is that we do not have the freedom to adjust the gas density or temperature or radiation as we please. (Recall that coronal models, to pick an example, do not include any dynamics and often treat the optical depth of the corona as a free parameter.)

Secondly, the model is essentially parameter-free. Given the binary parameters $M, i$ and $r_{\text {out }}$, the model makes a unique prediction of the flow configuration of the gas, and the spectrum of the radiation, as a function of $\dot{m}$. The model does involve a second parameter, the transition radius $r_{t r}$, but in principle this is a function of $\dot{m}$ (Figure 6 ). At low $\dot{m}, r_{t r}$ has a relatively large value determined by the nature of the mass transfer from the companion star (see the discussion in $\S 2$ ). When $\dot{m}=\dot{m}_{\text {crit }}, r_{t r}$ begins to decrease; this decrease is calculated self-consistently in the model. Finally, at high $\dot{m}$ there is no transition radius since the thin disk comes down to the marginally stable orbit. The model is thus quite well-constrained and does not allow much room for adjustment. The model does involve two important microscopic parameters, $\alpha$ and $\beta$, which parameterize the viscosity and the magnetic field strength (plus a third parameter $\delta$ which does not influence any of the results presented here). We have not treated $\alpha$ and $\beta$ as adjustable constants but rather assigned to them what we consider their most natural values $(\beta=0.5, \alpha \approx(1-\beta) / 2=0.25)$. We anticipate that with improved data in the future it may be possible to fit $\alpha$ and $\beta$. Hopefully, they are universal constants which once fitted on one source can be applied to any other source.

The main results obtained in this paper are the following:

1. The luminosities and spectra that we calculate in the low state agree well with observations published in the literature. For instance, when $\dot{m}$ approaches $\dot{m}_{\text {crit }}$, we obtain luminosities of a few percent of Eddington and hard spectra with photon indices $\sim 1.4-1.5$, which is consistent with the data (e.g. Nowak 1995). One interesting result is that we find the maximum $\dot{m}$ in the low state to be $\dot{m}_{\text {crit }} \sim 1.3 \alpha^{2}$ whereas the preliminary work of 
Narayan (1996) gave a scaling $\dot{m}_{\text {crit }} \sim 0.3 \alpha^{2}$. Also, we find the luminosity of the critical model to be $L_{\text {crit }} / L_{E d d} \sim 0.4 \alpha^{2}$ instead of $\sim 0.03 \alpha^{2}$. As a result of the new scalings, we can now explain the luminosities observed in the low state with a reasonable value of $\alpha \sim 0.25$ instead of $\alpha \sim 1$ required with the old scaling. The change appears to be related to improved global dynamics and better treatment of Compton-scattering in this paper (and in NBM) compared to Narayan (1996).

2. A major success of the model is the fact that it provides a natural explanation for one of the most dramatic features of BHXBs, namely the low-high state transition. As mentioned above, the ADAF which dominates the physics of the low state can exist only for $\dot{m}<\dot{m}_{\text {crit }}$. Once $\dot{m}$ crosses $\dot{m}_{\text {crit }}$, most of the gas in the hot flow settles into a thin accretion disk, with a weak optically thin corona above it. The spectrum therefore switches quite rapidly from the hard power-law spectrum characteristic of the low state to a soft spectrum which is dominated by the modified blackbody disk component. The intermediate spectral stages in this transition are shown in Figure 7, and these correspond quite well with the intermediate state which has been observed between the low and the high state (e.g. E94, Belloni et al. 1996, Mendez \& van der Klis 1997). Since the low-high transition occurs over a relatively narrow range of $\dot{m}$, the total luminosity of the system stays nearly constant. Furthermore, the X-ray spectrum displays an interesting pivoting behavior around $\sim 10 \mathrm{keV}$. These features have been seen in Nova Muscae (E94, §4) and Cyg X-1 (Zhang et al. 1997), and are convincingly explained in the model.

3. The model predicts that, in the high state, as $\dot{m}$ increases the mass in the corona decreases (Figure 9) and correspondingly the fraction of the flux in the hard tail goes down. This is confirmed by observations of Nova Muscae 1991 (Figure 13a). If the model is correct, this should be a universal feature of BHXBs in the high state, but we are not aware if the anti-correlation between the ultrasoft bump and the hard tail has been seen in other systems in the high state.

In $\S 4$, we present a quantitative comparison between the theoretical predictions of the model and the extensive observations of the outburst of Nova Muscae 1991 carried out by E94. The system parameters are well-known for this binary ( $\$ 4.1$, Table 1$)$. Using a very simple model for the mapping between the mass accretion rate $\dot{m}$, the transition radius $r_{t r}$ and the time of observation (equation 4-1), we present in $\S \S 4.3,4.4$ a detailed comparison of the models and the data. We use the theoretical model to predict the spectrum of Nova Muscae as a function of time and we compare the predictions against the data. The results are very encouraging (Figures 12-14). Not only is there a near-perfect qualitative agreement with the main features of the X-ray and $\gamma$-ray lightcurves, it even appears that the model is close to being a quantitatively predictive theory. Considering the level of self-consistency 
we have achieved and the relatively few parameters used in the comparison we find the results quite encouraging.

We also present in this paper a model of the very high state. The model is based on an idea pioneered by Haardt \& Maraschi (1991), namely that a part of the viscous energy released in the thin accretion disk may be input directly into the corona. We incorporate this possibility via an additional parameter $\eta(\S 3.5)$ and we compute model spectra as a function of $\dot{m}$ and $\eta$ (Figure 11). The results are not unreasonable. However, in contrast to the quiescent, low, intermediate and high states, where our model is robust and physically consistent, the proposal we make for the very high state is ad hoc. In fact, we include the very high state in this paper mainly for reasons of completeness, particularly for the comparisons in $\S 4$.

The modeling techniques we have used in this paper involve a number of improvements over previous versions of the model which were developed for applications to quiescent SXTs (NMY, NBM, Hameury et al. 1997). (1) By means of the parameter $f_{a v}$ (equation 2-2) we allow for the back reaction of radiative losses on the global dynamical solution of the ADAF. (2) Following the work of Nakamura et al. (1997) we have included energy advection in the thermal balance equation of the electrons (equation 2-7 and Appendix A). This allows us to compute a more accurate temperature profile for the electrons. (3) We have significantly enhanced the manner in which we model the coupling between the thin disk and the ADAF, taking care to include Compton reflection and the iron fluorescence line (§2.3). (4) We have also included pair processes in the ADAF and the corona (§2.4 and Appendix B), though we agree with the conclusion of Björnsson et al. (1996), and Kusunose \& Mineshige (1996) that pairs are not important.

Despite these enhancements to the model, several further improvements are possible. One long standing uncertainty in the model is the process by which material in the thin disk evaporates into the corona. A mechanism suggested by Meyer \& Meyer-Hofmeister (1994) is surface heating via electron conduction from the corona, while Honma (1996) suggested turbulent heat transport from the inner regions of the ADAF. Whatever the mechanism, one would like to be able to compute the evaporation rate from basic physical principles rather than fix $r_{t r}$ empirically.

Two simple changes would substantially improve our modeling of the thin disk. Instead of assuming that the disk emission is a pure blackbody, we could allow for graybody effects associated with electron scattering in the upper layers of the disk. This will have the effect of shifting the disk spectrum to higher energies (e.g. Shimura \& Takahara 1995). Secondly, we need to include vertical flaring so as to improve our model of the X-ray irradiation of the disk. We believe this will significantly reduce the discrepancy in the predicted optical/UV 
flux of Nova Muscae discussed in $§ 4.4$.

The models presented here are still based on Newtonian physics, and an obvious next step is to incorporate fully relativistic global flow solutions in Kerr geometry. Such solutions have been calculated by Abramowicz et al. (1996), Peitz \& Appl (1997) and Gammie \& Popham (1997). Apart from being more consistent, these solutions will allow us the opportunity to study the role of the spin parameter $a$ of the black hole. The effect of this parameter on spectra of ADAFs is at present completely unexplored. It is also necessary to include in the radiative transfer relativistic effects such as gravitational redshift, ray bending and Doppler boosts due to the motion of the gas. Titarchuk et al. $(1996,1997)$ have shown that the bulk motion of the gas as it flows into the black hole can have an important effect on the high energy end of the spectrum. This effect is not included in our present models.

So far we have not addressed the question of source variability, but the model makes several predictions in this regard. Though ADAFs are to some degree rotationally supported, the radial velocity reaches a significant fraction of free-fall. Using the self-similar solution of Narayan \& Yi (1994) the viscous timescale can be estimated to be $t_{v} \sim R / v_{r} \sim 0.025\left(M / 10 M_{\odot}\right)(0.25 / \alpha)(r / 10)^{3 / 2}$ s. The dynamical timescale is even shorter, $t_{d} \sim R / c_{s} \sim 0.003\left(M / 10 M_{\odot}\right)(r / 10)^{3 / 2}$ s. Thus, ADAFs can in principle produce very rapid variability, as shown by Manmoto et al. (1996). In comparison, the characteristic timescales associated with a thin accretion disk are significantly longer, especially when $r_{t r}$ is large. We thus expect short time scale variability to be strongly correlated with the power-law component of the emission, as seems to be observed (Nowak 1995).

Interactions between the hot inner ADAF and the cool outer thin disk at or near the transition radius can be an additional source of variability on longer timescales (Mendez \& van der Klis 1997). In this case, the characteristic time will be a multiple of the Keplerian rotation period at $r_{t r}, t_{K}=2 \pi R / v_{K} \sim 1\left(M / 10 M_{\odot}\right)(r / 100)^{3 / 2} \mathrm{~s}$, and it is conceivable that the variations might be quasi-periodic. The model thus makes a very specific prediction. During the low-high state transition, any characteristic frequency in the variability spectrum (e.g. QPOs) should be low when the spectrum is hard ( $r_{t r}$ is large) and high when the spectrum is soft $\left(r_{t r}\right.$ is small). The detection of $\sim 0.1 \mathrm{~Hz}$ QPO in the power spectra of BHXBs in the low state and $\sim 10 \mathrm{~Hz}$ QPO in the high state (Nowak 1995, Cui et al. 1997) is certainly very suggestive. If this behavior is confirmed as a general trend in BHXBs, then it would be a strong confirmation of the model.

The basic paradigm of our model, namely that the thin disk is limited to a large radius in the low state and quiescent state but comes close to the black hole in the high state and very high state, can also be tested through direct determination of $r_{t r}$. One method is 
by fitting the ultrasoft component in the spectrum to a multicolor blackbody (technically graybody). This was attempted in the case of Nova Muscae by E94. However, during the high-low state transition, which is when $r_{t r}$ is expected to vary, the disk emission peaks at energies below $1 \mathrm{keV}$, making the results rather uncertain. A more indirect, but equally promising way of detecting the change in $r_{t r}$ during this transition is through observations of Compton reflection of hard X-rays off the surface of the disk, and by measuring the strength and spectral width of the iron fluorescence line. The physical width of the line is directly correlated with the rotational velocity at the inner edge of the disk which is $\propto r_{t r}^{1 / 2}$, while the equivalent width contains information about the solid angle subtended by the thin disk as viewed from the radiating zones of the ADAF. We have already included Compton reflection in the model, and we compute some of the properties of the iron line. There is a clear qualitative prediction according to the model: both Compton reflection and the iron line equivalent width should become stronger as a BHXB proceeds from the low state to the high state. In order to make direct quantitative comparisons with the data we will need to use a more realistic iron line profile shape than we have done so far and take into account the 3-D shape of the accretion disk.

We conclude by clarifying a point of terminology. An ADAF is defined as an accretion flow where energy advection dominates over cooling. However, in the models with $\dot{m}>\dot{m}_{\text {crit }}$ described in this paper only $35 \%$ of the heat energy is advected (corresponding to $\left.f_{a v}=0.35\right)$ and the flows are not ADAFs in the strict definition of the term. These flows nevertheless belong to the same solution branch which at lower values of $\dot{m}$ are truly advection-dominated with $f_{a v} \rightarrow 1$. For this reason, we feel it is appropriate to label them as ADAFs.

We thank K. Ebisawa, S. Kitamoto and J. Orosz for providing data and for helpful discussions. This work was supported in part by NSF grant AST 9423209 and NASA grant NAG 5-2837. A. A. E. was supported by a National Science Foundation Graduate Research Fellowship, and partial support for J. E. M. was provided by the Smithsonian Institution Scholarly Studies Program. 


\section{A. Energy Balance Equation for Electrons}

Electrons in the accretion flow must satisfy the general energy conservation requirement

$$
\rho T_{e} v \frac{d s_{e}}{d R}=q^{i e}+\delta q^{+}-q^{-},
$$

where $s_{e}$ is the entropy of the electrons per unit total gas mass, $q^{i e}$ is the Coulomb energy transfer rate from ions to electrons per unit volume, $\delta q^{+}$is the fraction of the viscous energy dissipation that goes directly into the electrons, and $q^{-}$is the cooling rate per unit volume. In our previous work we set the left hand side of this equation to zero and required that the heating and cooling rates balance at all radii. However, Nakamura et al. (1997) have made the important point that energy advection by electrons may dominate under some circumstances. We therefore consider the full equation in this work.

We know that

$$
T_{e} d s_{e}=d u_{e}+P_{e} d\left(\frac{1}{\rho}\right),
$$

where $u_{e}$ is the internal energy of the electrons per unit mass and $P_{e}$ is the electron pressure. The quantities on the right side of this equation are in general easy to compute. However, our case is complicated by the fact that we need to consider a mixture of gas and magnetic fields. If gas pressure contributes a constant fraction $\beta$ to the total pressure in the flow, $P_{t o t}$ can be written as

$$
P_{t o t}=\frac{\rho k T_{i}}{\mu_{i} m_{u}}+\frac{\rho k T_{e}}{\mu_{e} m_{u}}+\frac{B^{2}}{24 \pi}=\frac{\rho k T_{i}}{\beta \mu_{i} m_{u}}+\frac{\rho k T_{e}}{\beta \mu_{e} m_{u}} .
$$

It seems natural then to denote the two resulting terms as the effective ion and electron pressures, so that $P_{e}=\rho k T_{e} /\left(\beta \mu_{e} m_{u}\right)$.

The internal energy of the gas is a sum of the ion and electron internal energies plus the magnetic energy density:

$$
u=\frac{3}{2} \frac{k T_{i}}{\mu_{i} m_{u}}+a\left(T_{e}\right) \frac{k T_{e}}{\mu_{e} m_{u}}+\frac{B^{2}}{8 \pi \rho}=\frac{6-3 \beta}{2 \beta} \frac{k T_{i}}{\mu_{i} m_{u}}+\left[\frac{3(1-\beta)}{\beta}+a\left(T_{e}\right)\right] \frac{k T_{e}}{\mu_{e} m_{u}},
$$

where the coefficient $a\left(T_{e}\right)$ varies from $3 / 2$ in the case of a non-relativistic electron gas, to 3 for fully relativistic electrons. The general expression for $a$ as a function of the dimensionless electron temperature $\theta_{e}=k T_{e} / m_{e} c^{2}$ was derived by Chandrasekhar (1939, equation [236]) to be

$$
a\left(\theta_{e}\right)=\frac{1}{\theta_{e}}\left(\frac{3 K_{3}\left(1 / \theta_{e}\right)+K_{1}\left(1 / \theta_{e}\right)}{4 K_{2}\left(1 / \theta_{e}\right)}-1\right) .
$$


Note that the ion thermal energy in these flows never exceeds $10 \%$ of the ion rest mass, so that the corresponding coefficient for the ions is always $\sim 3 / 2$.

As we have done for the pressure, the right hand side of equation (A4) is naturally divided into two terms, the ion and electron internal energies:

$$
u_{i}=\frac{6-3 \beta}{2 \beta} \frac{k T_{i}}{\mu_{i} m_{u}}, \quad \text { and } \quad u_{e}=\left[\frac{3(1-\beta)}{\beta}+a\left(T_{e}\right)\right] \frac{k T_{e}}{\mu_{e} m_{u}} .
$$

In this interpretation, $u_{e}$ and $u_{i}$ are again "effective" quantities, which include contributions from the particles as well as the associated magnetic field. Note that the contribution of the magnetic field to the internal energy of each particle species is proportional to the contribution of these particles to the total pressure, a natural choice in our model where the ratio of the magnetic to gas pressure is fixed.

Having defined $P_{e}$ and $u_{e}$, we can now compute $d s_{e} / d R$. Then equation (A1) becomes

$$
\frac{\rho v k}{\mu_{e} m_{u}}\left[\frac{3(1-\beta)}{\beta}+a\left(T_{e}\right)+T_{e} \frac{d a}{d T_{e}}\right] \frac{d T_{e}}{d R}+\frac{\rho k T_{e}}{\beta \mu_{e} m_{u}}\left[\frac{d v}{d R}+\frac{2 v}{R}\right]=q^{i e}+\delta q^{+}-q^{-},
$$

where we have used mass conservation to replace the radial derivative of gas density, $d \rho / d R$, by terms that depend on the radial velocity $v$ and its derivative $d v / d R$. Finally, to obtain the electron energy equation for each shell of the ADAF, we multiply both sides of the equation above by the appropriate shell volume.

\section{B. Electron-Positron Pair Production and Annihilation}

Local pair equilibrium requires that the pair creation rate is balanced by pair annihilation, as expressed in equation (2-12). The pair annihilation rate per unit volume for a thermal distribution of electrons and positrons was derived by Svensson (1982a)

$$
\left(\dot{n}_{+}\right)_{\mathrm{ann}}=\frac{\pi c r_{e}^{2} n_{+} n_{-}}{1+2 \theta_{e}^{2} / \ln \left(1.12 \theta_{e}+1.3\right)},
$$

where $n_{+}$and $n_{-}$are the positron and electron number densities respectively (in the main body of the paper the electron number density is denoted by $n_{e}$ ).

Pair production in relativistic plasmas occurs through several interactions. In this paper we consider electron-electron $(e e)$, photon-electron $(\gamma e)$, photon-proton $(\gamma p)$, and photon-photon $(\gamma \gamma)$, collisions (note that "electron" here means either electron or positron). Thus

$$
\left(\dot{n}_{+}\right)_{\text {prod }}=\left(\dot{n}_{+}\right)_{e e}+\left(\dot{n}_{+}\right)_{\gamma e}+\left(\dot{n}_{+}\right)_{\gamma p}+\left(\dot{n}_{+}\right)_{\gamma \gamma}
$$


We ignore electron-proton interactions, which were shown by Svensson (1982b, hereafter $\mathrm{S} 82 \mathrm{~b})$ to be less efficient than ee collisions by a factor of $\sim 10$. Though Svensson's result was derived for a one-temperature plasma where $\theta_{p}=\theta_{e}$, it is probably still valid in our case, since the protons in the ADAF are not relativistic.

The electron-electron pair production rate per unit volume in the non-relativistic and extreme relativistic limits is (White \& Lightman 1989)

$$
\left(\dot{n}_{+}\right)_{e e}=c r_{e}^{2}\left(n_{+}+n_{-}\right)^{2} \begin{cases}2 \times 10^{-4} \theta_{e}^{3 / 2} \exp \left(-2 / \theta_{e}\right)\left(1+0.015 \theta_{e}\right), & \theta_{e} \ll 1 \\ (112 / 27 \pi) \alpha_{f}^{2}\left(\ln \theta_{e}\right)^{3}\left(1+0.058 / \theta_{e}\right)^{-1}, & \theta_{e} \gg 1\end{cases}
$$

where $\alpha_{f}=1 / 137.04$ is the fine structure constant. To join the two limiting expressions smoothly at $\theta_{e} \sim 1$, we use $\left(\dot{n}_{+}\right)_{e e}=\left(\dot{n}_{+}\right)_{e e}^{\theta_{e} \ll 1} \times e^{-\theta_{e}}+\left(\dot{n}_{+}\right)_{e e}^{\theta_{e} \gg 1} \times e^{-1 / \theta_{e}^{3}}$.

The pair production rate via photon-electron collisions is given by the double integral $(\mathrm{S} 82 \mathrm{~b})$

$$
\left(\dot{n}_{+}\right)_{\gamma e}=\frac{c\left(n_{+}+n_{-}\right)}{2 K_{2}\left(1 / \theta_{e}\right)} \int_{0}^{\infty} d x \frac{n_{\gamma}(x)}{x^{2}} \int_{4}^{\infty} d y y \sigma_{\gamma e}(y) \exp \left[-(x / y+y / x) / 2 \theta_{e}\right],
$$

where $n_{\gamma}(x)$ is the number density of photons with energy $x=h \nu / m_{e} c^{2}$. Because of the exponential factor, the greatest contribution to the second integral comes from photons with energies $x \sim y>4$. Using the numerical fit for the cross section $\sigma_{\gamma e}(y)$ given in Stepney \& Guilbert (1983) we numerically evaluate the two integrals at each point in the accretion flow.

Since the proton temperature in ADAFs is always below $10^{12} \mathrm{~K}$, protons are never relativistic $\left(\theta_{p} \lesssim 0.09\right)$ and the photon-proton pair production rate can be simply written as $(\mathrm{S} 82 \mathrm{~b})$

$$
\left(\dot{n}_{+}\right)_{\gamma p}=c n_{p} \int_{2}^{\infty} d x n_{\gamma}(x) \sigma_{\gamma p}(x)
$$

where we again adopt the numerical fit to the cross section $\sigma_{\gamma p}(x)$ from Stepney \& Guilbert (1983), and integrate over the photons with energies above $2 m_{e} c^{2}$.

Finally, the photon-photon pair production rate is given by $\mathrm{S} 82 \mathrm{~b}$

$$
\left(\dot{n}_{+}\right)_{\gamma \gamma}=\frac{c}{2} \int_{0}^{\infty} d x n_{\gamma}(x) \int_{1 / x}^{\infty} d y n_{\gamma}(y)\left\langle\sigma_{\gamma \gamma}(x y, \phi)\right\rangle_{\phi}
$$

where $\left\langle\sigma_{\gamma \gamma}(x y, \phi)\right\rangle_{\phi}$ is the interaction cross section averaged over the photon-photon interaction angle $\phi$, taken from Gould \& Schreder (1966). The factor of $1 / 2$ takes care of double counting of interacting photon pairs. Note that only the pairs with $x y \geq 1$ contribute to pair production. 
We now to rewrite the above rate equations in terms of the pair fraction $z=n_{+} / n_{p}$. For simplicity, we assume that the accreting gas contains only ionized hydrogen, so that $n_{p}=\rho / m_{p}$. Then we can write $n_{+}=z n_{p}$ and $n_{-}=n_{p}+n_{+}=(z+1) n_{p}$. With these definitions, equation (2-12) becomes

$$
n_{p} z(1+z) g_{\mathrm{ann}}=n_{p}(1+2 z)^{2} g_{e e}+n_{p}(1+2 z) g_{\gamma e}+n_{p} g_{\gamma p}+g_{\gamma \gamma}
$$

where the coefficients $g_{\text {ann }}, g_{e e}, g_{\gamma e}, g_{\gamma p}$, and $g_{\gamma \gamma}$ contain all the detailed physics described above. This new equation is quadratic in $z$ and can be readily solved at each point in the advection flow, given the gas density, electron temperature and the radiation field. Because of the dependence on the latter, the final solution has to be obtained iteratively, since newly created electrons and positrons contribute to the radiative processes, and thus help create more pairs. 


\section{REFERENCES}

Abramowicz, M. A., Chen, X., Grantham, M., Lasota, J.-P. 1996, ApJ, 471, 762

Abramowicz, M. A., Chen, X., Kato, S., Lasota, J. P., \& Regev, O. 1995, ApJ, 438, L37

Abramowicz, M. A., Czerny, B., Lasota, J. P., \& Szuszkiewicz, E. 1988, ApJ, 332, 646

Balbus, S. A. \& Hawley, J. F. 1991, ApJ, 376, 214

Begelman, M. C. 1978, MNRAS, 184, 53

Begelman, M. C. \& Chiueh, T. 1988,ApJ, 332, 872

Belloni, T., Mendez, M., van der Klis, M., Hasinger, G., Lewin, W. H. G., van Paradijs, J. 1996, ApJ, 472, L107

Björnsson, G., Abramowicz, M. A., Chen, X., Lasota, J.-P. 1996, ApJ, 467, 99

Brandt, S., Castro-Tirado, A. J., Lund, N., Dremin, V., Lapshov, I., Sunyaev, R. A. 1992, A\&A, 254, L39

Cannizzo, J. K. 1993, in Accretion Disks in Compact Stellar Systems, ed. J. C. Wheeler, Singapore: World Scientific Publishing, 6

Casares, J., Martin, E. L., Charles, P. A., Molaro, P., Rebolo, R. 1997, New Astr., 1, 299

Chandrasekhar, S. 1939, in Introduction to the Study of Stellar Structure

Chen, X. 1995, MNRAS, 275, 641

Chen, X., Abramowicz, M. A., Lasota, J. P. 1997, ApJ, 476, 61

Chen, X., Abramowicz, M. A., Lasota, J. P., Narayan, R., Yi, I. 1995, ApJ, 443, L61

Clayton, D. D. 1983, Principles of Stellar Evolution and Nucleosynthesis, U. of Chicago Press

Cui, W., Heindl, W. A., Rothschild, R. E., Zhang, S. N., Jahoda, K., Focke, W. 1997, ApJ, 474, L57

Ebisawa, K. et al. 1994, PASJ, 46, 375 (E94)

Ebisawa, K., Ueda, Y., Inoue, H., Tanaka, Y., White, N. E. 1996, ApJ, 467, 419

Eggleton, P. P. 1983, ApJ, 268, 368

Esin, A. A. 1997, ApJ, 482, in press (astro-ph/9701039)

Fabian, A. C. \& Rees, M. J. 1995, MNRAS, 277, 55

Field, G. B. \& Rogers, R. D. 1993, ApJ, 403, 94

Frank, J., King, A., \& Raine, D. 1992, Accretion Power in Astrophysics (Cambridge, UK: Cambridge University press) 
Gammie, C. \& Popham, R. 1997, submitted to ApJ

George, I. M. \& Fabian, A. C. 1991, MNRAS, 249, 352

Gierliński, M., Zdziarski, A. A., Done, C., Johnson, W. N., Ebisawa, K., Ueda, Y., Haardt, F., Phlips, B. F. 1997, MNRAS, in press (astro-ph/9610156)

Gilfanov, M. et al. 1993, A\&A Suppl. Ser., 97, 303

Goldwurm, A. et al. 1992, ApJ, 389, L79

Gould, R. J. \& Schreder, G. P. 1966, Phys. Rev., 155, 1404

Grebenev, S. A. et al. 1991, 17, 413

Haardt, F. \& Maraschi, L. 1991, ApJ, 380, L51

Hameury, J.-M., Lasota, J.-P., McClintock, J. E., \& Narayan, R. 1997, submitted to ApJ

Hawley, J. F. \& Balbus, S. A. 1996, in Physics of Accretion Disks, eds. S. Kato et al., Gordon and Breach Sci. Publ., 273

Hawley, J. F., Gammie, C. F., Balbus, S. A. 1995, ApJ, 440, 742

Hawley, J. F., Gammie, C. F., Balbus, S. A. 1996, ApJ, 444, 690

Honma, F. 1996, PASJ, 48, 77

Huang, M. \& Wheeler, J. C. 1989, ApJ, 343, 229

Igumenshchev, I. V., Chen, X., Abramowicz, M. A. 1996, MNRAS, 278, 236

Jaroszyński, M. \& Kurpiewski, A. 1997, A\&A, in press (astro-ph/9705044)

Kato, S., Abramowicz, M. \& Chen, X. 1996, PASJ, 48, 67

Katz, J. I. 1977, ApJ, 215, 265

Kitamoto, S., Tsunemi, H., Miyamoto, S., \& Hayashida, K. 1992, ApJ, 394, 609

Kurfess, J. D. 1996, A\&A Suppl. Ser., 120, 5

Kusunose, M. \& Mineshige, S. 1996, ApJ, 468, 330

Lasota, J. P., Abramowicz, M. A., Chen, X., Krolik, J., Narayan, R., \& Yi, I. 1996a, ApJ, $462,142 \mathrm{~L}$

Lasota, J. P., Narayan, R., \& Yi, I. 1996b, A\&A, 314, 813

Liang, E. 1997, Phys. Rep., in press

Lightman, A. P. \& White, T. R. 1988, ApJ, 335, 57

Lund, N. 1993, A\&A Suppl. Ser., 97, 289

Mahadevan, R. 1997, ApJ, 477, 585 
Manmoto, T., Takeuchi, M., Mineshige, S., Matsumoto, R., Negoro, H. 1996, ApJ, 464, L135

McClintock, J. E., Horne, K., \& Remillard, R. A. 1995, ApJ, 442, 358

Melia, F. \& Misra, R. 1993, ApJ, 411, 797

Mendez, M. \& van der Klis, M. 1997, ApJ, 479, 926

Meyer, F. \& Meyer-Hofmeister, E. 1994, A\&A, 288, 175

Mineshige, S. 1996, PASJ, 48, 93

Mineshige, S. \& Wheeler, J. C. 1989, ApJ, 343, 241

Miyamoto, S., Kimura, K., Kitamoto, S., Dotani, T., \& Ebisawa, K. 1991, ApJ, 383, 784

Morrison, R. \& McCammon, D. 1983, ApJ, 270, 119

Nakamura, K. E., Kusunose, M., Matsumoto, R., \& Kato, S. 1997, to appear in PASJ

Nandra, K. \& Pounds, K. A. 1994, MNRAS, 268, 405

Narayan, R. 1997, in Proc. IAU Colloq. 163 on Accretion Phenomena \& Related Outflows, ASP Conf. Series, eds. D. T. Wickramasinghe et al., in press (astro-ph/9611113)

Narayan, R. 1996, ApJ, 462, 136

Narayan, R., Barret, D., \& McClintock, J. E. 1997a, ApJ, in press (NBM)

Narayan, R., Garcia, M. R. \& McClintock, J. E. 1997b, ApJ, 478, 79

Narayan, R., Kato, S. \& Honma, F. 1997c, ApJ, 476, 49

Narayan, R., McClintock, J. E., \& Yi, I. 1996, ApJ, 457, 821 (NMY)

Narayan, R. \& Yi, I. 1994, ApJ, 428, L13

Narayan, R. \& Yi, I. 1995a, ApJ, 444, 231

Narayan, R. \& Yi, I. 1995b, ApJ, 452, 710

Narayan, R., Yi, I., \& Mahadevan, R. 1995, Nature, 374, 623

Novikov, I. D. \& Thorne, K. S. 1973, in Black Holes, ed. DeWitt, C. and B. (Gordon \& Breach, NY), 343

Nowak, M. A. 1995, PASP, 107, 1207

Orosz, J. A., Bailyn, C. D., Remillard, R. A., McClintock, J. E., \& Foltz, C. B. 1994, ApJ, 436,848

Orosz, J. A., Bailyn, C. D., McClintock, J. E., \& Remillard, R. A. 1996, ApJ, 468, 380

Orosz, J. A., Remillard, R. A., Bailyn, C. D., \& McClintock, J. E. 1997, ApJ, 478, 83 
Peitz, J. \& Appl, S. 1997, MNRAS, in press (astro-ph/9612205)

Piran, T. 1978, ApJ, 221, 652

Popper, D. M. 1980, ARA\&A, 18, 115

Poutanen, J. \& Svensson, R. 1996, ApJ, 470, 249

Rees, M. J., Phinney, E. S., Begelman, M. C., Blandford, R. D. 1982, Nature, 275, 17

Reynolds, C. S., Di Matteo, T., Fabian, A. C., Hwang, U., Canizares, C. R. 1996, MNRAS, 283, L111

Sakimoto, P. J. \& Coroniti, F. V. 1981, ApJ, 247, 19

Shahbaz, T., Naylor, T. \& Charles, P. A. 1997, MNRAS, 285, 607

Shakura, N. I. \& Sunyaev, R. A. 1973, A\&A, 24, 337

Shapiro, S. L., Lightman, A. P., \& Eardley, D. M. 1976, ApJ, 204, 187 (SLE)

Shimura, T. \& Takahara, F. 1995, ApJ, 445, 780

Shrader, C. R. \& Gonzalez-Riestra, R. 1993, A\&A, 276, 373

Stepney, S., \& Guilbert, P. W. 1983, MNRAS, 204, 1269

Sunyaev, R. A. \& Titarchuk, L. G. 1980, A\&A, 86, 121

Sunyaev, R. A. et al. 1993, A\&A, 280, L1

Svensson, R. 1982a, ApJ, 258, 321

Svensson, R. 1982b, ApJ, 258, 335 (S82b)

Svensson, R. \& Zdziarski, A. A 1994, ApJ, 436, 599

Tanaka, Y. et al. 1995, Nature, 375, 659

Tanaka, Y. \& Lewin, W. H. G. 1995, X-ray Binaries, eds. W. H. G. Lewin et al., Cambridge Univ. Press., 126

Tanaka, Y. \& Shibazaki, N. 1996, ARA\&A, 34, 607

Titarchuk, L. G., Mastichiadis, A. \& Kylafis, N. D. 1996, A\&A Suppl. Ser., 120, C171

Titarchuk, L. G., Mastichiadis, A. \& Kylafis, N. D. 1997, ApJ, in press (astro-ph/9702092)

van der Klis, M. 1994, ApJS, 92, 511

van Paradijs, J. \& McClintock, J. E. 1995, X-ray Binaries, eds. W. H. G. Lewin et al., Cambridge Univ. Press., 58

Vrtilek, S. D., Raymond, J. C., Garcia, M. R., Verbunt, F., Hasinger, G., Kurster, M. 1990, A\&A, 235, 162 
Wandel, A. \& Liang, E. 1991, ApJ, 380, 84

White, T. R. \& Lightman, A. P. 1989, 340, 1024

White, T. R. Lightman, A. P. \& Zdziarski, A. A. 1988, ApJ, 331, 939

Zhang, S. N., Cui, W., Harmon, B. A., Paciesas, W. S., Remillard, R. E., van Paradijs, J. 1997, ApJ, 477, L95 
Table 1. Standard parameter values

\begin{tabular}{ccccccc}
\hline \hline$M\left(M_{\odot}\right)$ & $i$ & $\log r_{\text {out }}$ & $\log r_{\text {tr }}{ }^{\mathrm{a}}$ & $\alpha$ & $\beta$ & $\delta$ \\
\hline 6 & $60^{\circ}$ & 4.9 & 3.9 & 0.25 & 0.5 & 0.001 \\
\hline
\end{tabular}

${ }^{a}$ in quiescence 


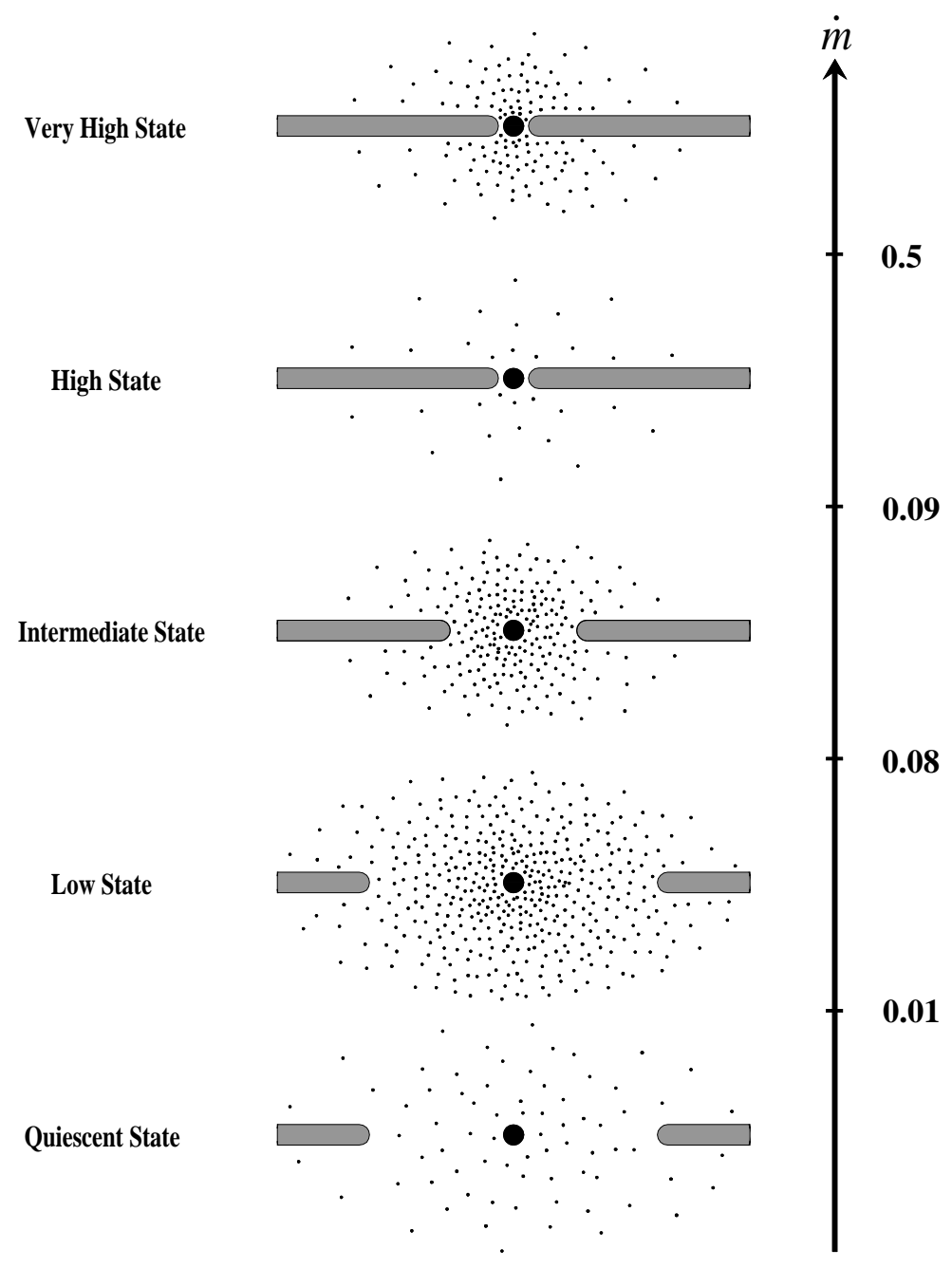

Fig. 1.- The configuration of the accretion flow in different spectral states shown schematically as a function of the total mass accretion rate $\dot{m}$. The ADAF is indicated by dots and the thin disk by the horizontal bars. The lowest horizontal panel shows the quiescent state which corresponds to a low mass accretion rate (and therefore, a low ADAF density) and a large transition radius. The next panel shows the low state, where the mass accretion rate is larger than in the quiescent state, but still below the critical value $\dot{m}_{c r i t} \sim 0.08$. In the intermediate state (the middle panel), $\dot{m}>\dot{m}_{\text {crit }}$ and the transition radius is smaller than in the quiescent/low state. In the high state, the thin disk extends down to the last stable orbit and the ADAF is confined to a low-density corona above the thin disk. Finally, in the very high state, we make the tentative proposal that the corona has a substantially larger $\dot{m}$ than in the high state. 


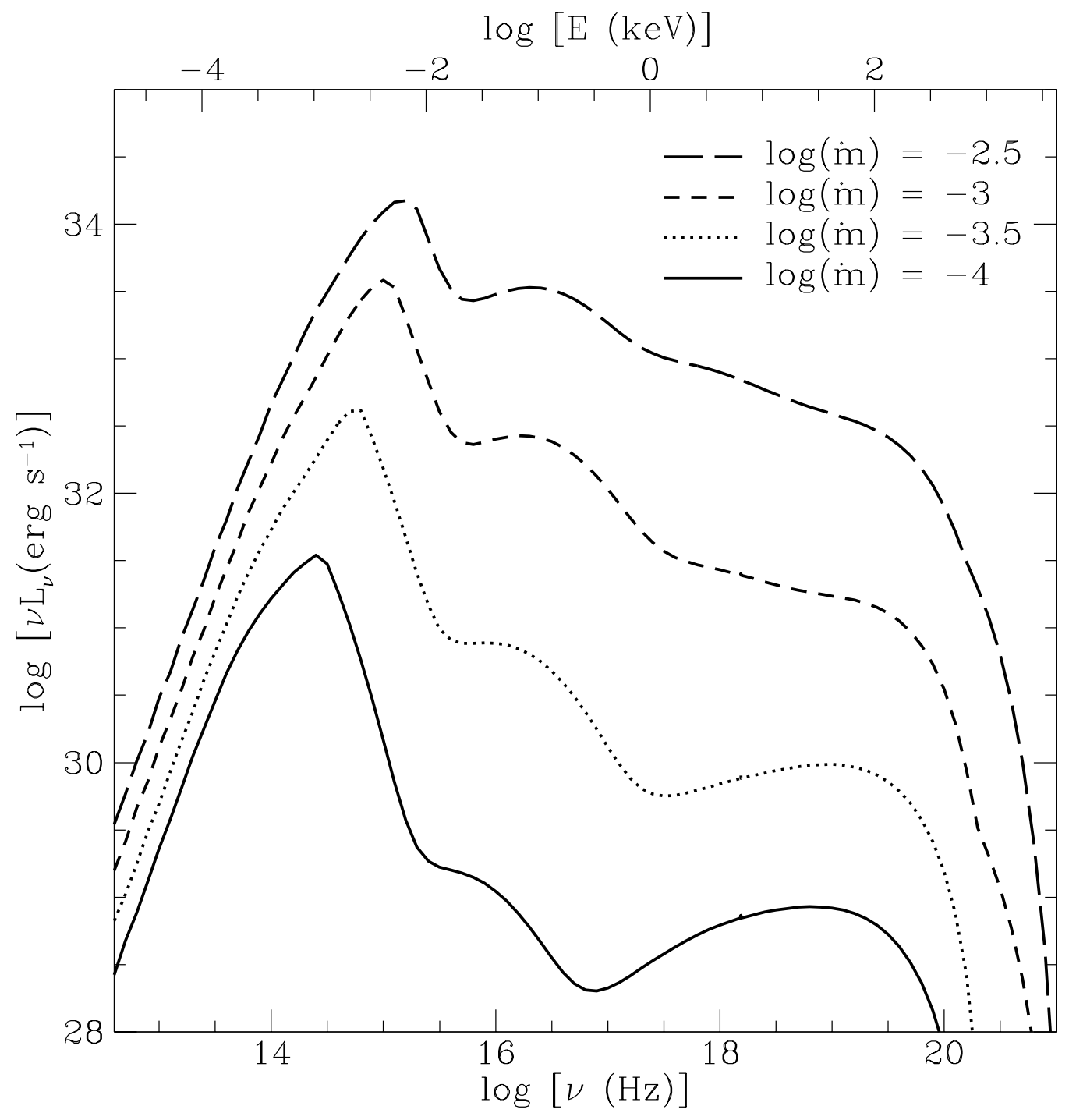

Fig. 2.- Quiescent state spectra for models with $M=6 M_{\odot}, \log \left(r_{t r}\right)=3.9, i=60^{\circ}$, $\alpha=0.25$, and $\beta=0.5$. The values of $\dot{m}$ are indicated on the plot. 


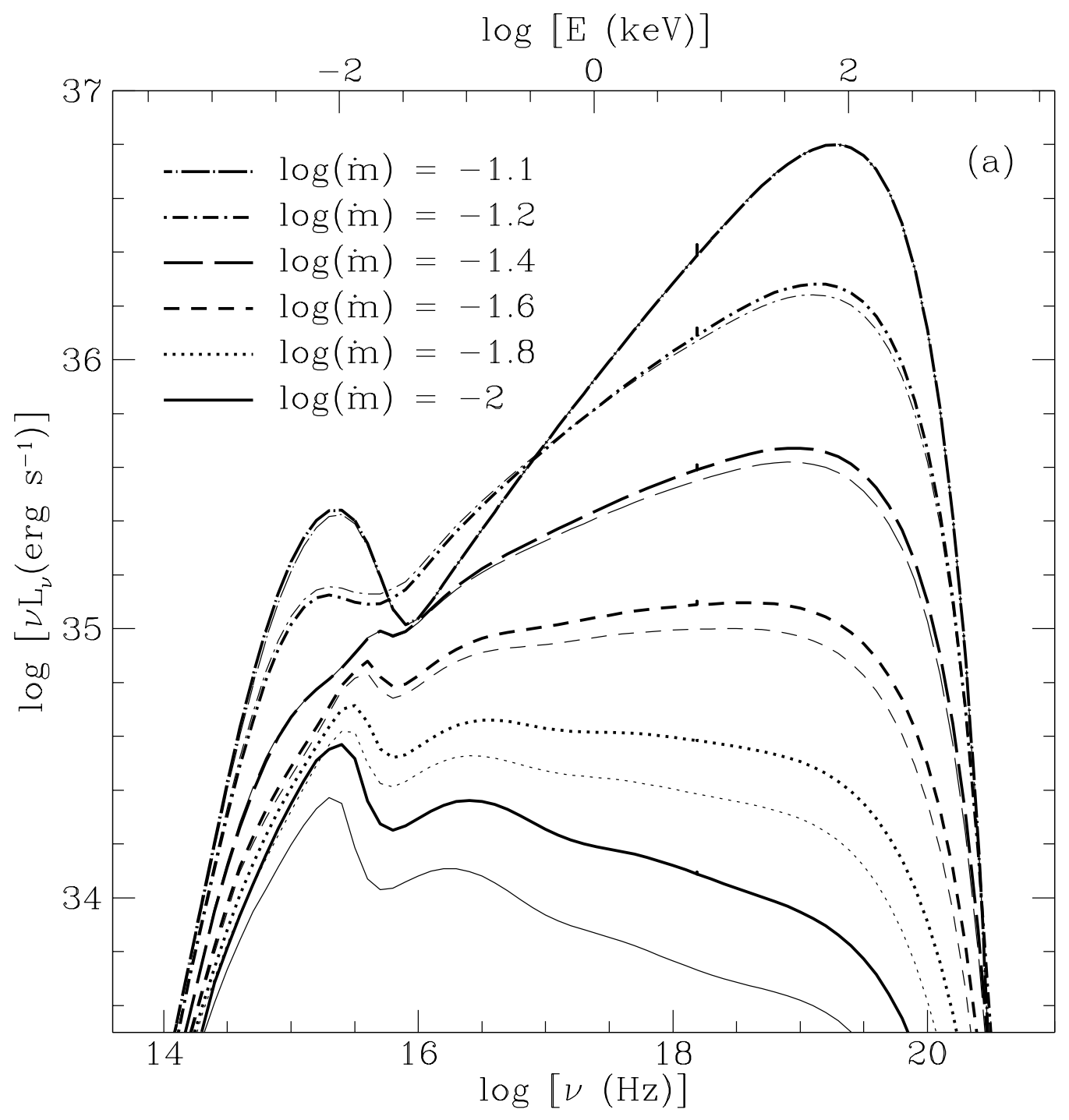

Fig. 3.- (a) Low state spectra for models with $M=6 M_{\odot}, \log \left(r_{t r}\right)=3.9, i=60^{\circ}, \alpha=0.25$, $\beta=0.5$. The values of $\dot{m}$ are indicated on the plot. The largest $\dot{m}$ is equal to the critical accretion rate $\dot{m}_{\text {crit }}$. Heavy lines show spectra computed with the electron advection term included; for comparison, thin lines show spectra of models where this term is omitted. 


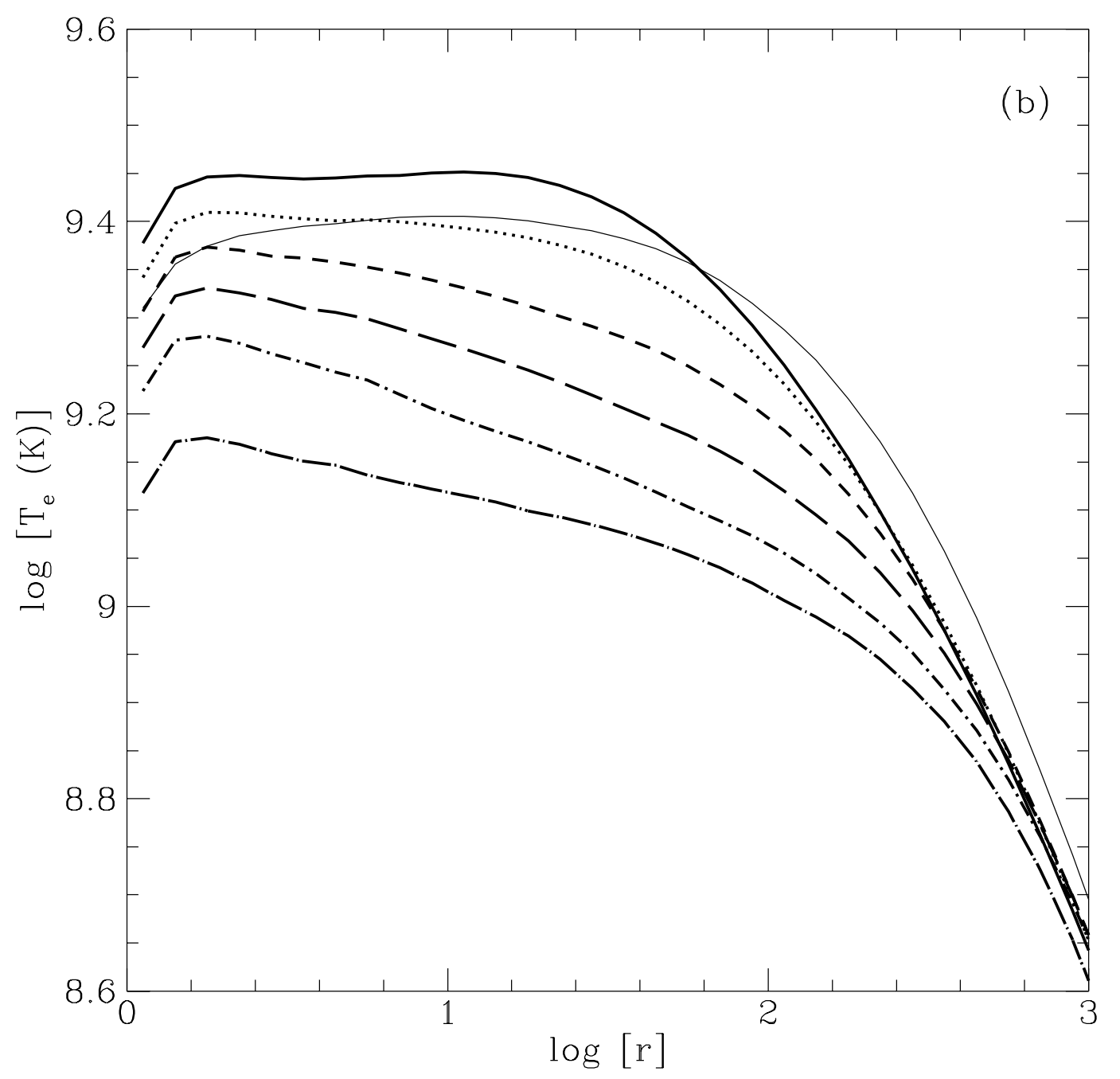

Fig. 3.- (b) Variation of electron temperature $T_{e}$ with radius $r$ for the models shown in (a). Note that the electron temperature decreases with increasing $\dot{m}$. For the model with $\log \dot{m}=-2$ (solid line), the temperature profile is computed both with (heavy line) and without (thin line) the electron advection term. 


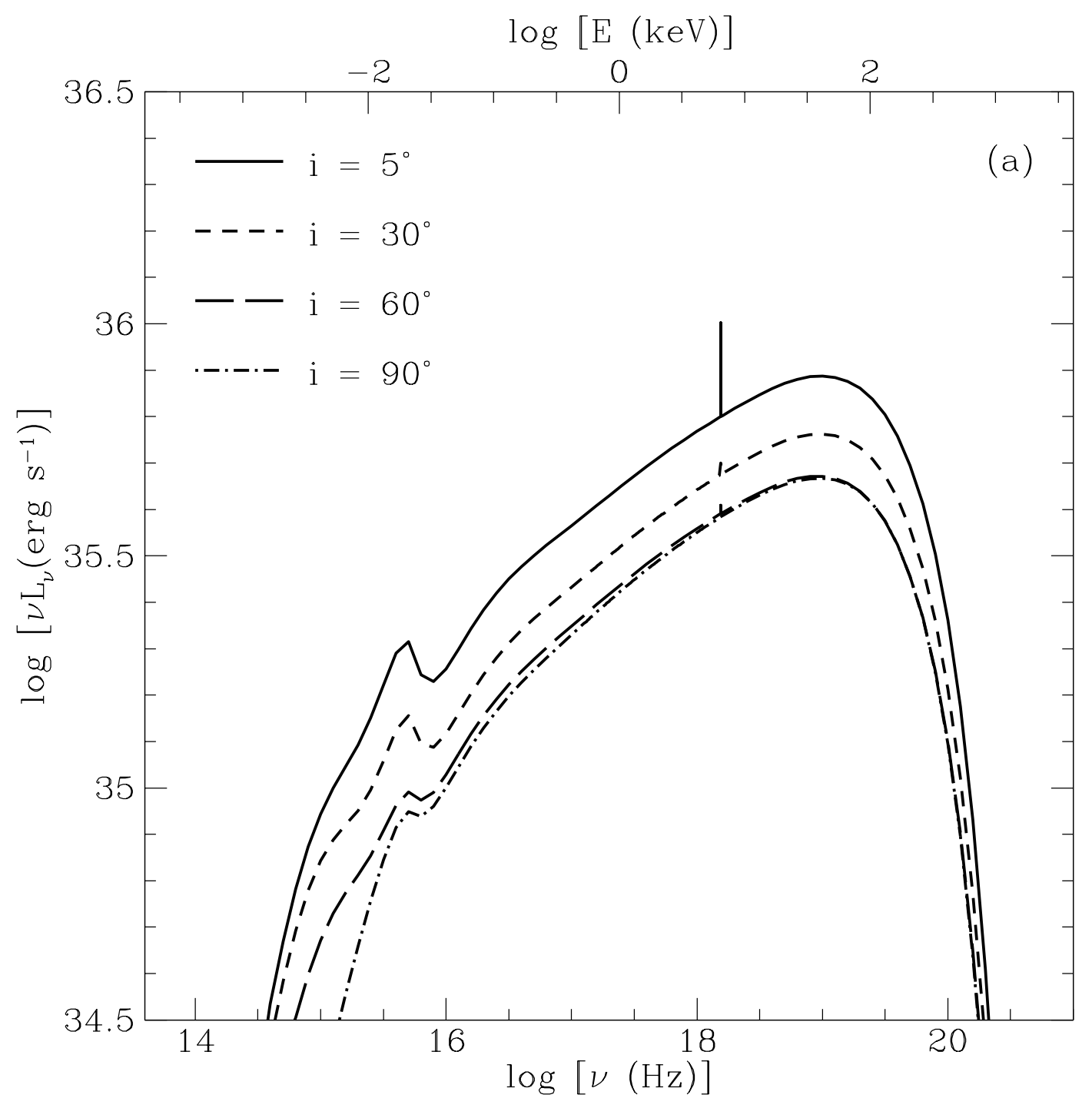

Fig. 4.- (a) Spectra of a low state model with $M=6 M_{\odot}, \log \left(r_{t r}\right)=3.9, i=60^{\circ}, \alpha=0.25$, $\beta=0.5$, and $\log \dot{m}=-1.4$ viewed at different inclination angles. The feature at $6.4 \mathrm{keV}$ is the iron $\mathrm{K} \alpha$ line. Its width is proportional to $\sin i$, so the line is more prominent in face-on systems, though the equivalent width is independent of $i$. 


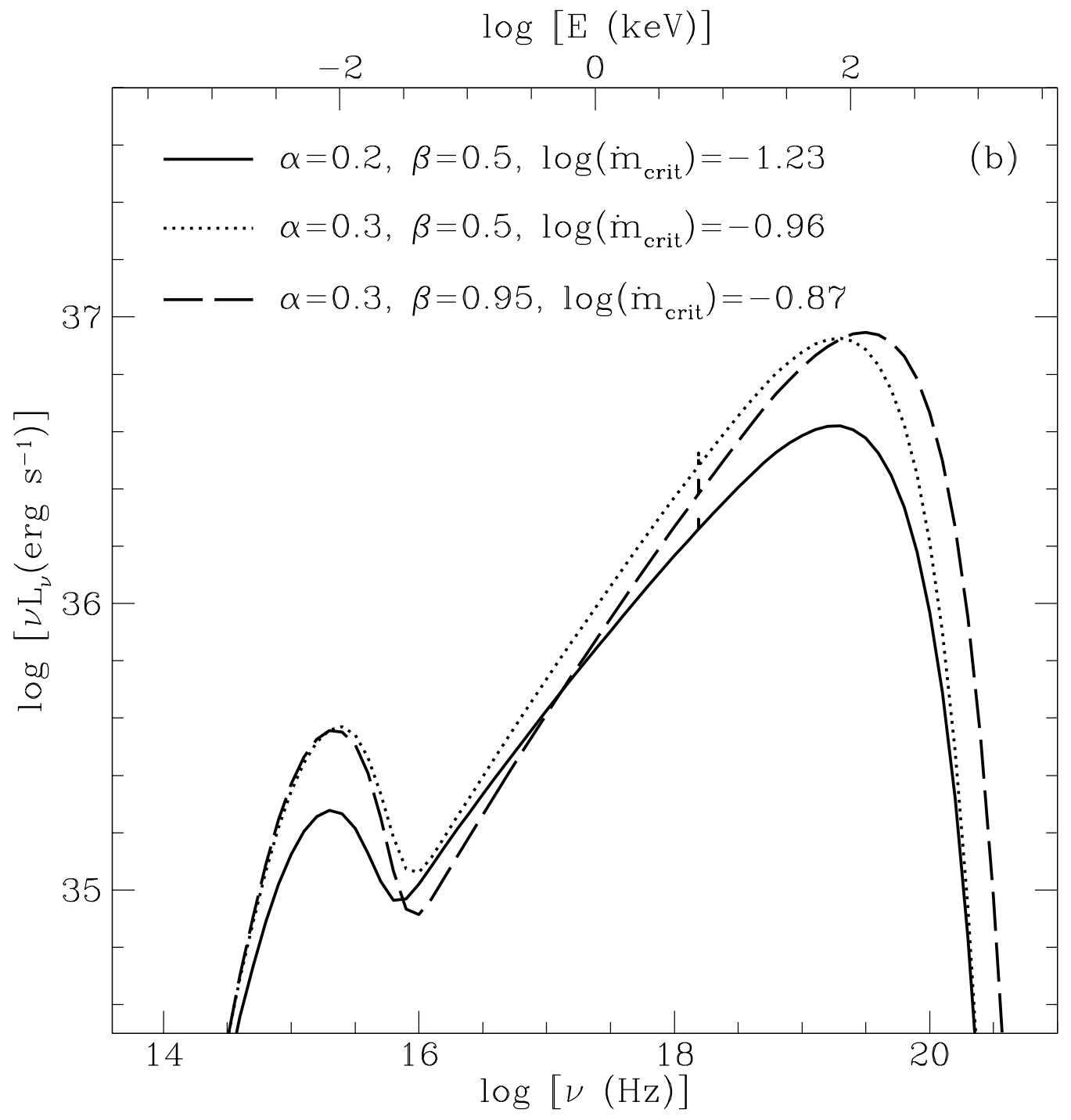

Fig. 4.- (b) Low state spectra for a model with $M=6 M_{\odot}, \log \left(r_{t r}\right)=3.9, i=60^{\circ}$, and values of $\alpha, \beta$, and $\dot{m}$ as shown on the figure. Each spectrum corresponds to a model with the critical mass accretion rate $\dot{m}_{\text {crit }}$. 


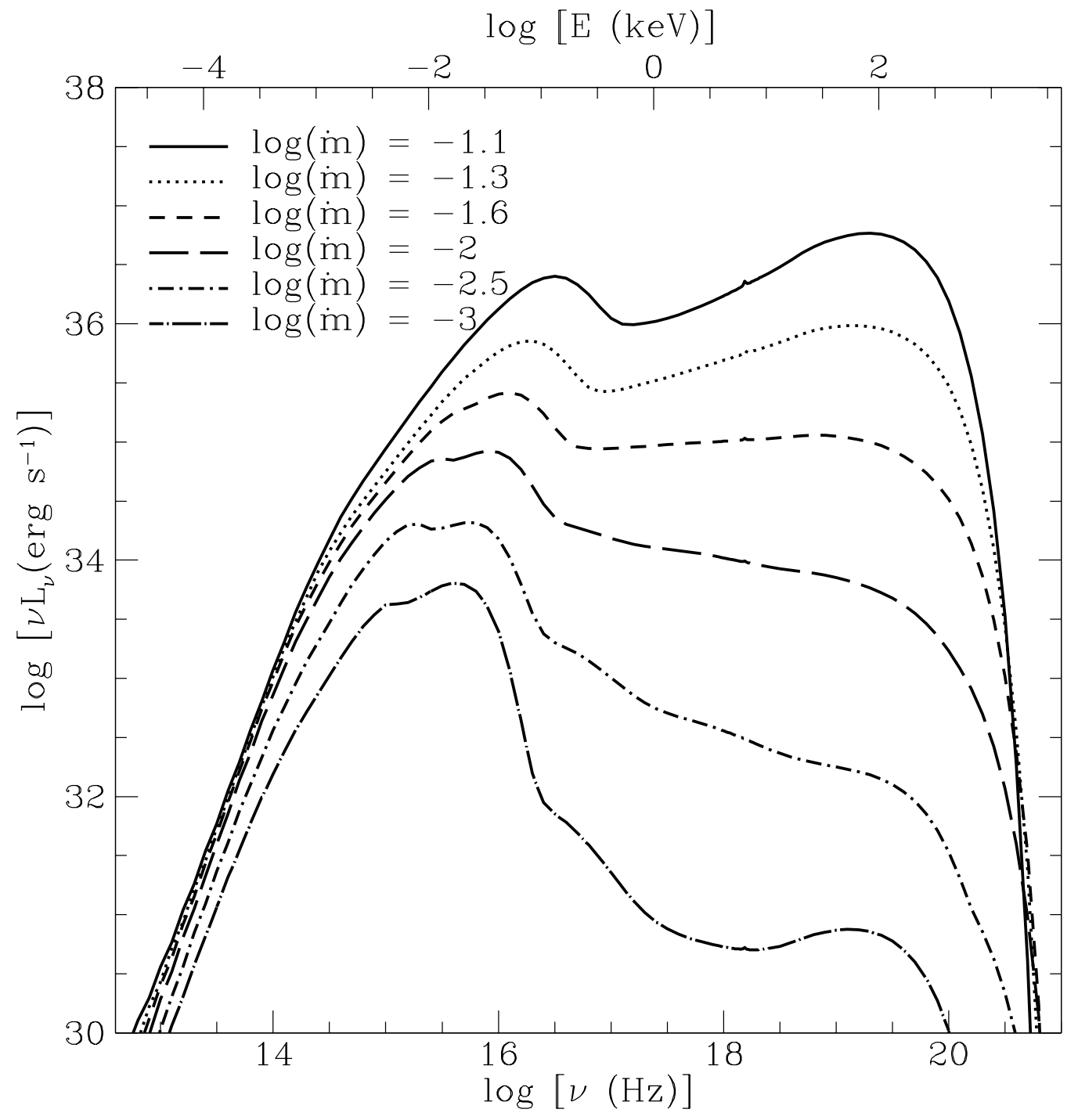

Fig. 5.- Low state and quiescent state spectra for a wind-fed system with $M=6 M_{\odot}$, $\log \left(r_{t r}\right)=2, i=60^{\circ}, \alpha=0.25, \beta=0.5$ and values of the accretion rate as shown on the figure. The spectra in the X-ray band are the same as for a system with a larger $r_{t r}$ (compare with Figure 2 and Figure 3). However in the optical and UV bands the thin disk emission now dominates over the synchrotron emission, and the soft peak moves from the optical into the UV and soft X-ray band. 

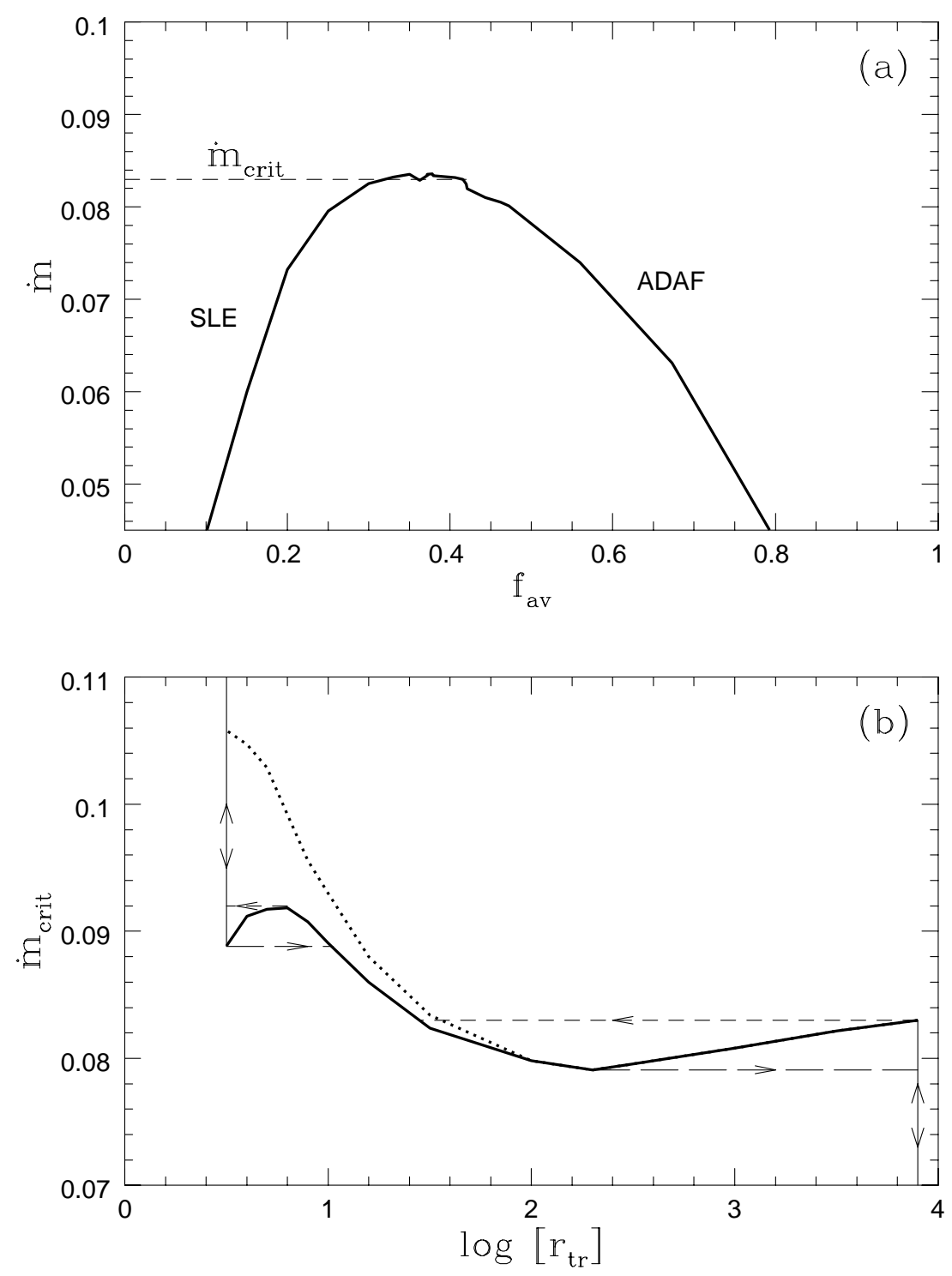

Fig. 6.- (a) $\dot{m}$ vs. $f_{a v}$ for a model with $M=6 M_{\odot} \alpha=0.25, \beta=0.5$, and $\log \left(r_{t r}\right)=3.9$. The maximum allowed mass accretion rate, $\dot{m}_{\text {crit }}$, corresponds to $f_{a v} \sim 0.35$. The models with $f_{a v}<0.35$ correspond to the thermally unstable SLE solutions, while the models with $f_{a v}>0.35$ are the advection-dominated solutions used in this paper. (b) The heavy solid line shows $\dot{m}_{c r i t}$ as a function of $r_{t r}$. Thin lines indicate possible tracks followed by a system during the rise phase (short-dashed line) and decay phase (long-dashed line) of an outburst. The dotted line shows $\dot{m}_{c r i t}\left(r_{t r}\right)$ computed under the assumption that half of the energy needed for evaporation of the thin disk material into the corona comes from the energy budget of the thin disk. 


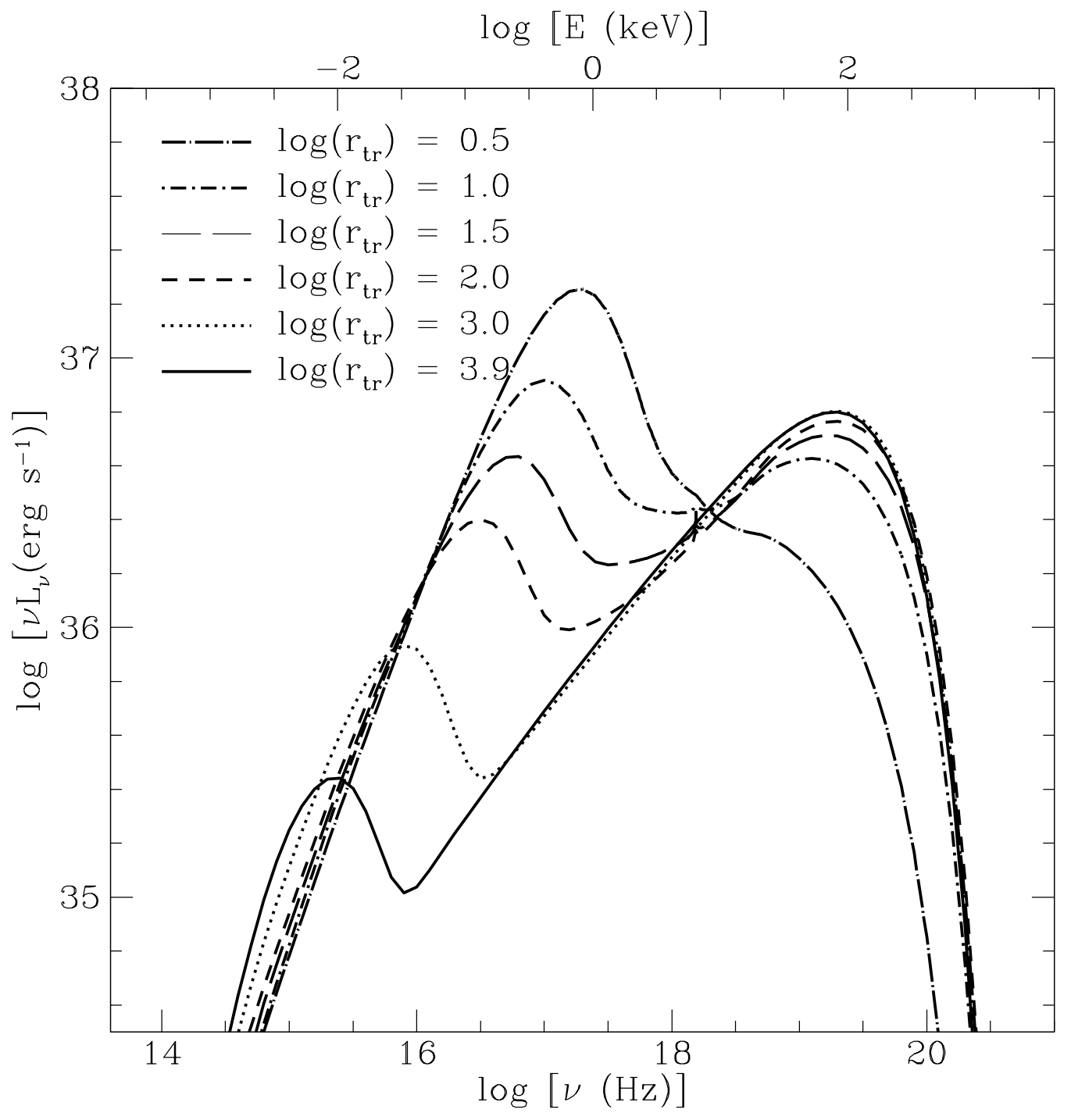

Fig. 7.- Spectra of intermediate state models with $M=6 M_{\odot}, i=60^{\circ}, \alpha=0.25, \beta=0.5$. Values of the transition radius are indicated on the figure; the accretion rate is taken to be the critical rate, $\dot{m}_{\text {crit }}\left(r_{t r}\right)$, shown in Figure $6 \mathrm{~b}$. Note that the spectra pivot around $10 \mathrm{keV}$. Note also the appearance of a reflection bump centered near $30 \mathrm{keV}$ in the spectra of models with $r_{t r} \leq 10^{2}$, and the broadening of the iron $\mathrm{K} \alpha$ line at $6.4 \mathrm{keV}$. 


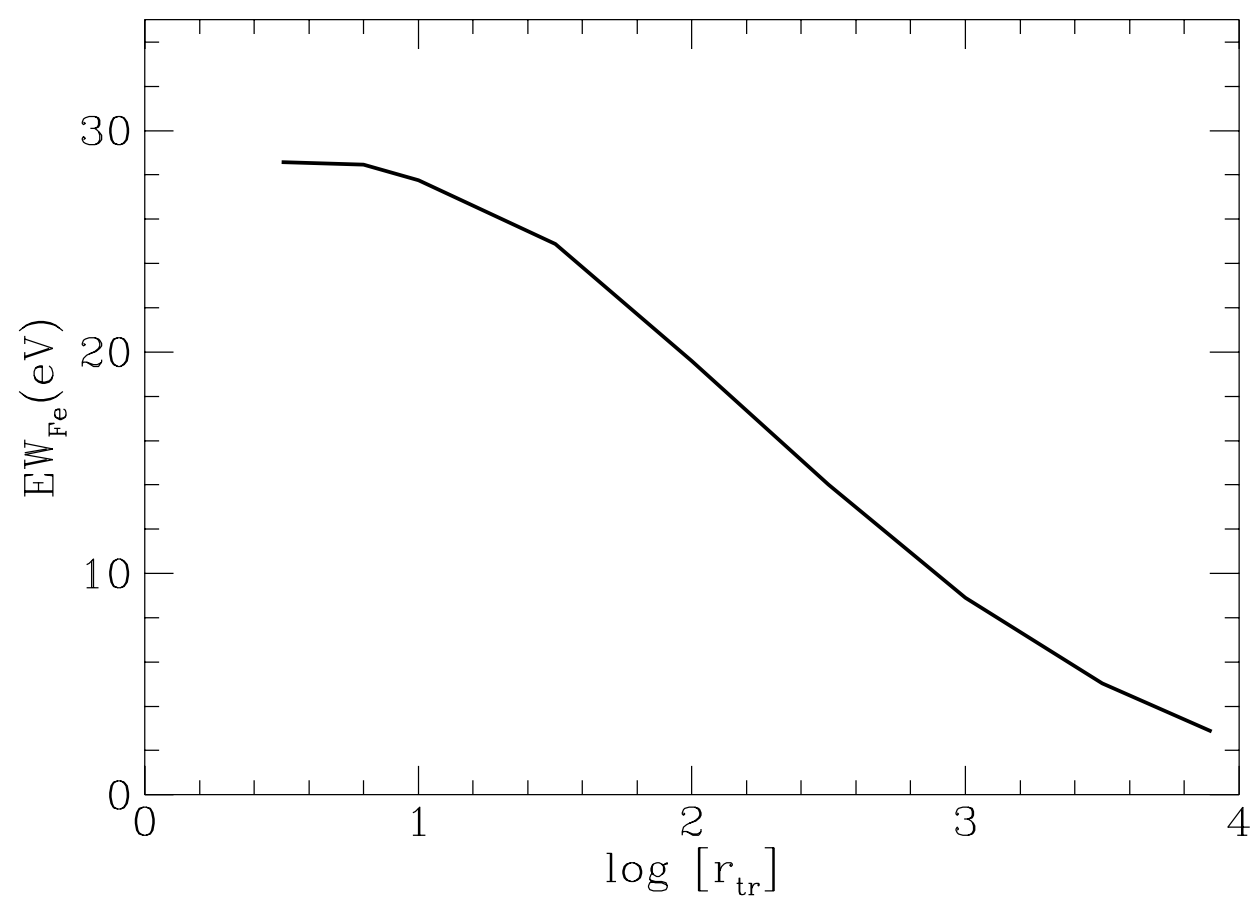

Fig. 8. - Variation of the iron $\mathrm{K} \alpha$ line equivalent width as a function of the transition radius $r_{t r}$ in the intermediate state models shown in Figure 7. 


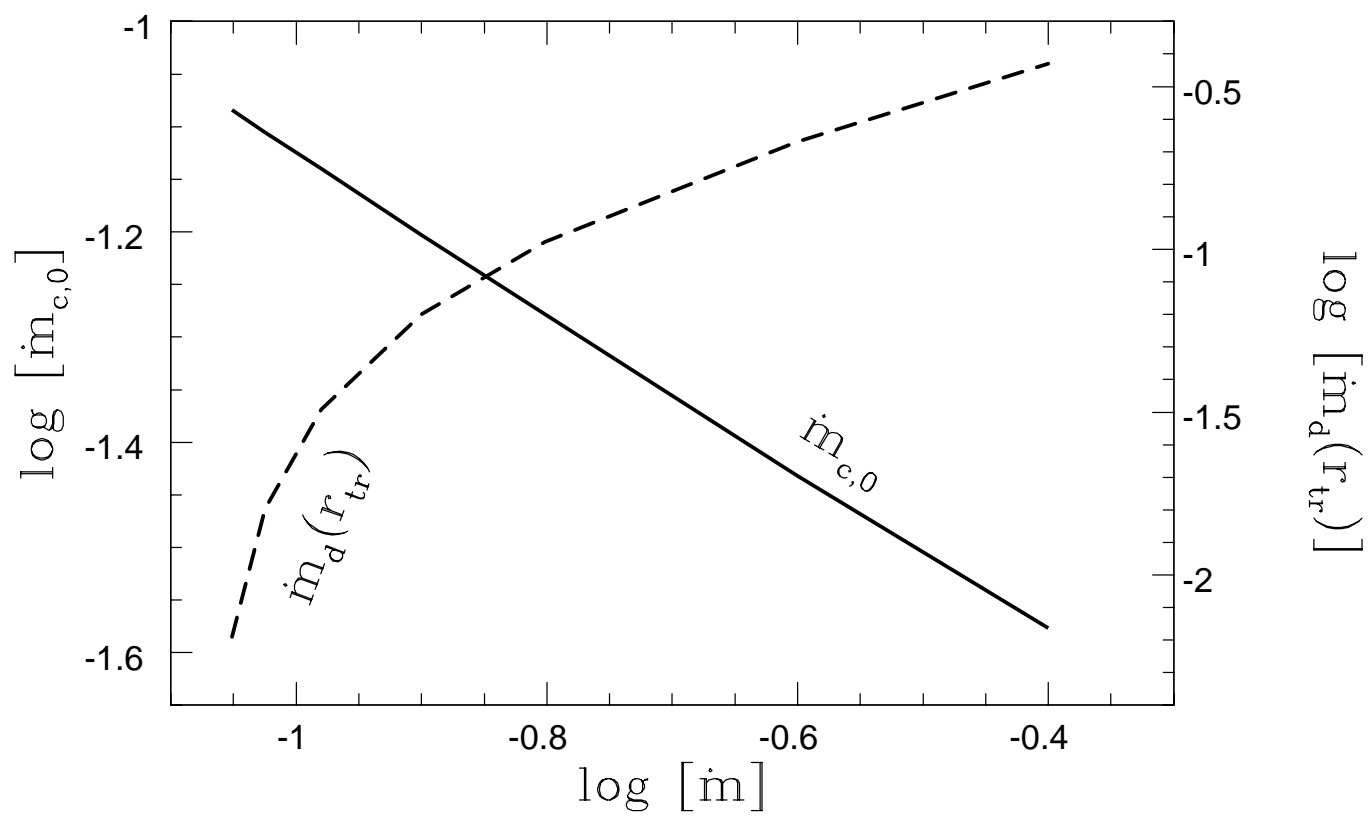

Fig. 9.- The solid line corresponds to the critical accretion rate in the corona, $\dot{m}_{c, 0}$, as a function of the total accretion rate, $\dot{m}$. The dashed line is the corresponding accretion rate in the thin disk at the last stable orbit, $\dot{m}_{d}(r=3)=\dot{m}-\dot{m}_{c, 0}$. Note that $\dot{m}_{c, 0}$ decreases with increasing $\dot{m}$. Thus the coronal emission becomes weaker with increasing total luminosity. 


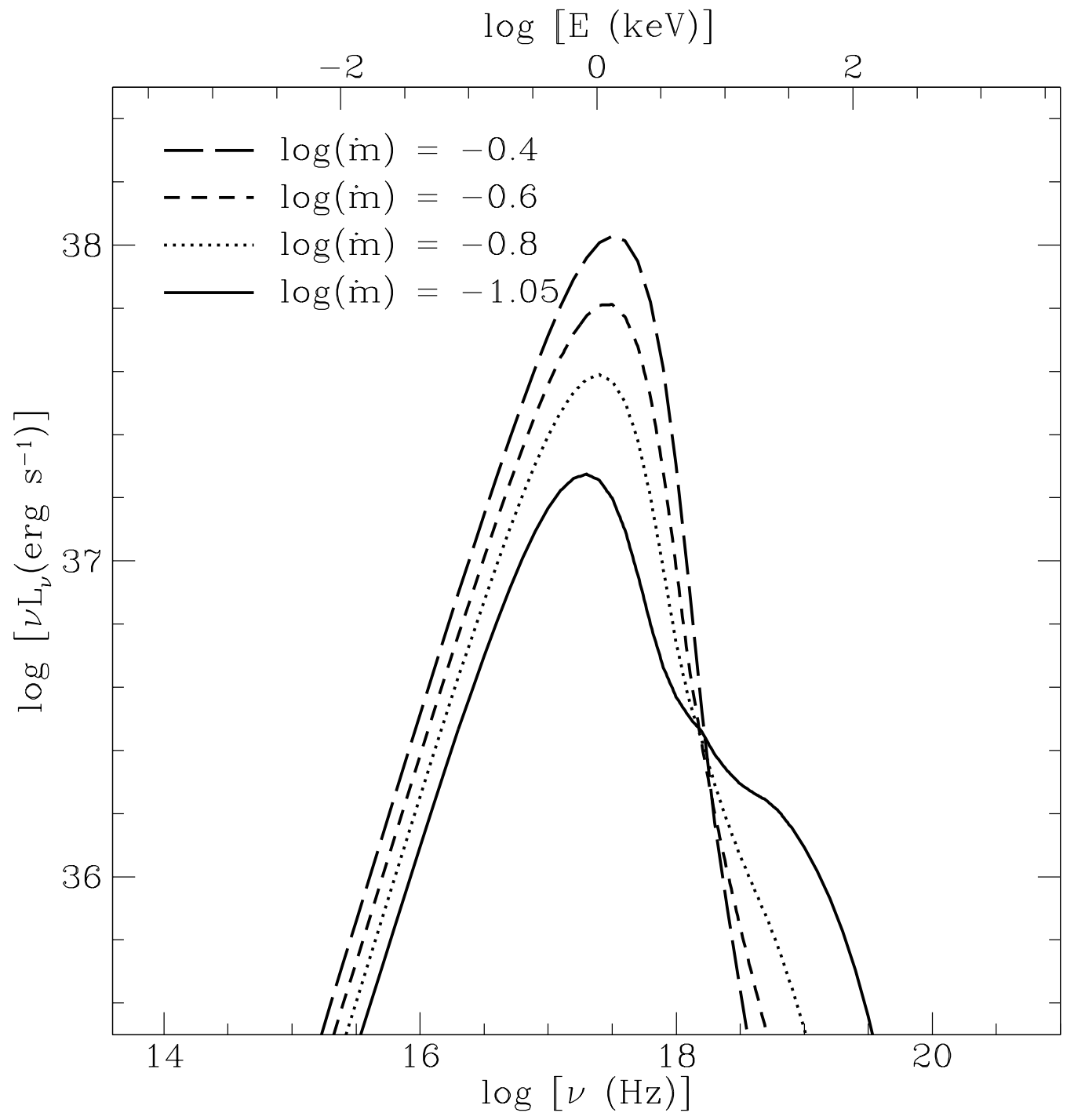

Fig. 10.- Spectra of high state models with $M=6 M_{\odot}, i=60^{\circ}, r_{t r}=3, \alpha=0.25, \beta=0.5$, and $\dot{m}$ as indicated on the figure. At higher $\dot{m}$, the corona contains less gas (see Figure 9 ), and consequently, the amount of high energy emission decreases. 


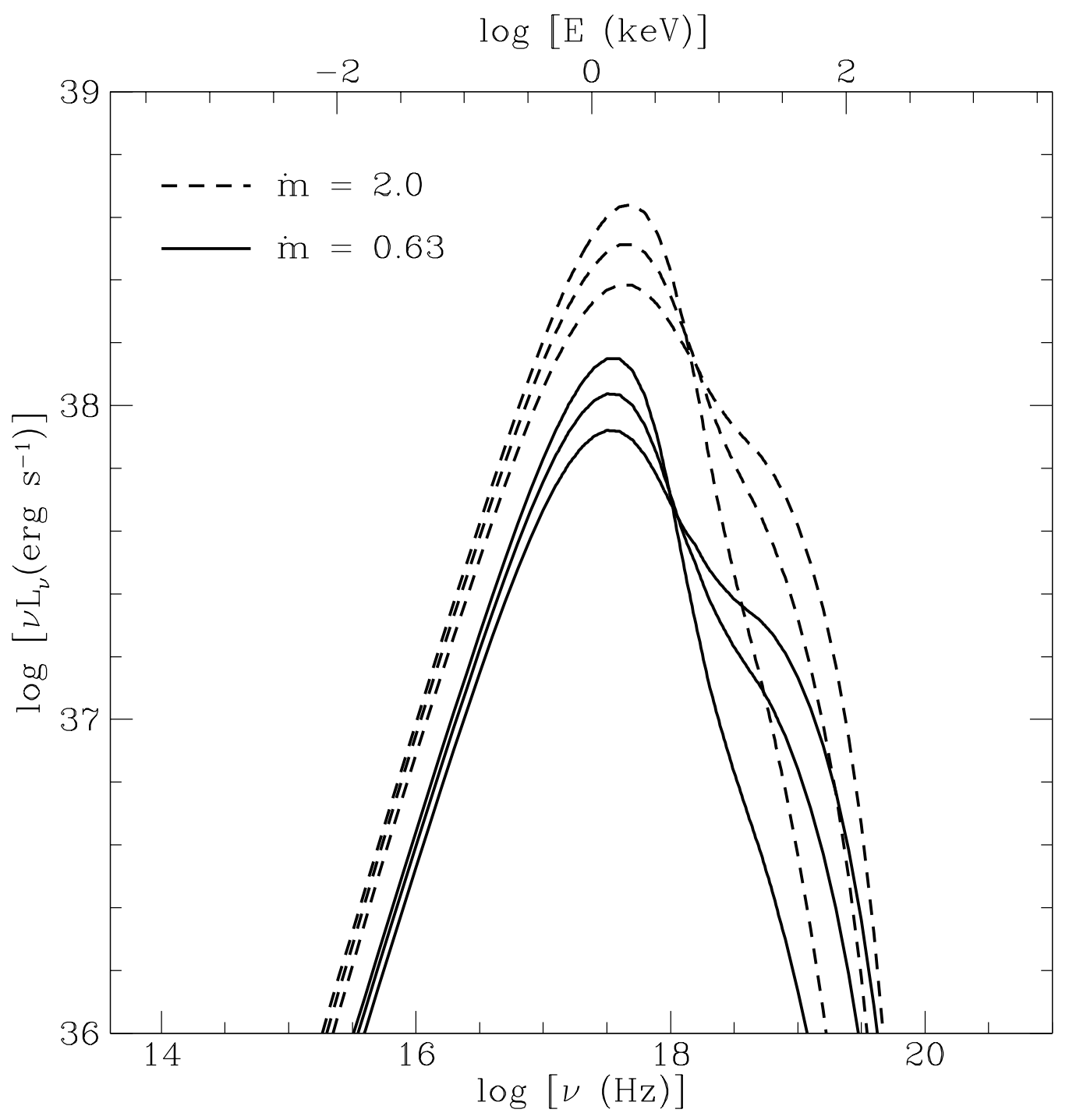

Fig. 11. - Spectra of very high state models with $M=6 M_{\odot}, i=60^{\circ}, r_{t r}=3, \alpha=0.25$, $\beta=0.5$. For each value of $\dot{m}$ three spectra are shown with $\eta=0.1,0.3,0.5$ in order of increasing flux at $10 \mathrm{keV}$. Note that with increasing $\eta$ the disk blackbody emission at $\sim 2$ keV decreases, but the high energy tail becomes more prominent. 


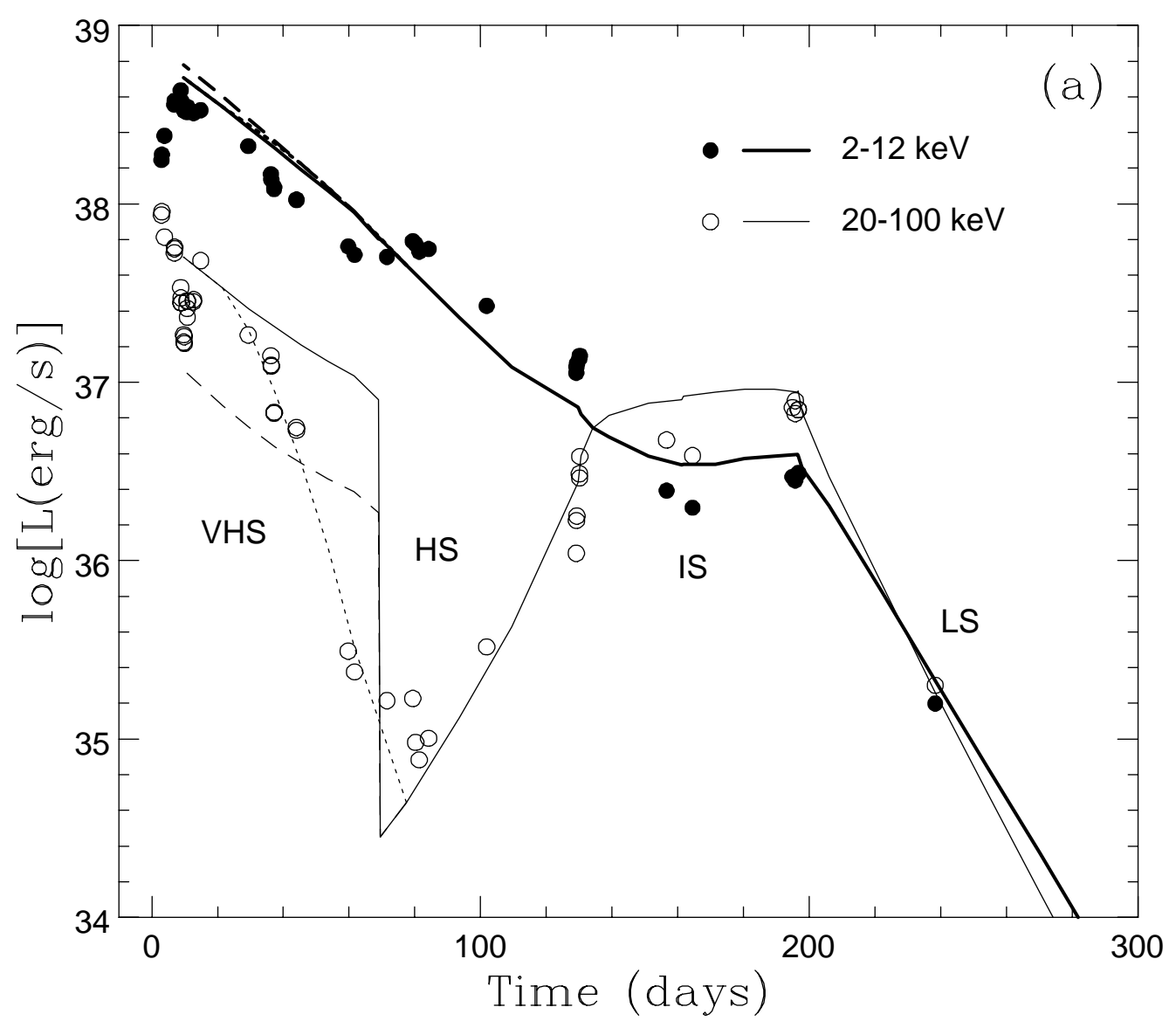

Fig. 12.- (a) X-ray and $\gamma$-ray light curves of Nova Muscae 1991. Filled and open circles are data from E94 corresponding to the $2-12 \mathrm{keV}$ and $20-100 \mathrm{keV}$ bands, respectively. The heavy and thin lines are the corresponding model predictions. The symbols VHS, HS, IS, LS correspond to the very high state, high state, intermediate state and low state respectively. In the very high state, the solid and dashed lines correspond to models with $\eta=0.3$ and 0.1 , while the dotted line corresponds to a model with variable $\eta$. 


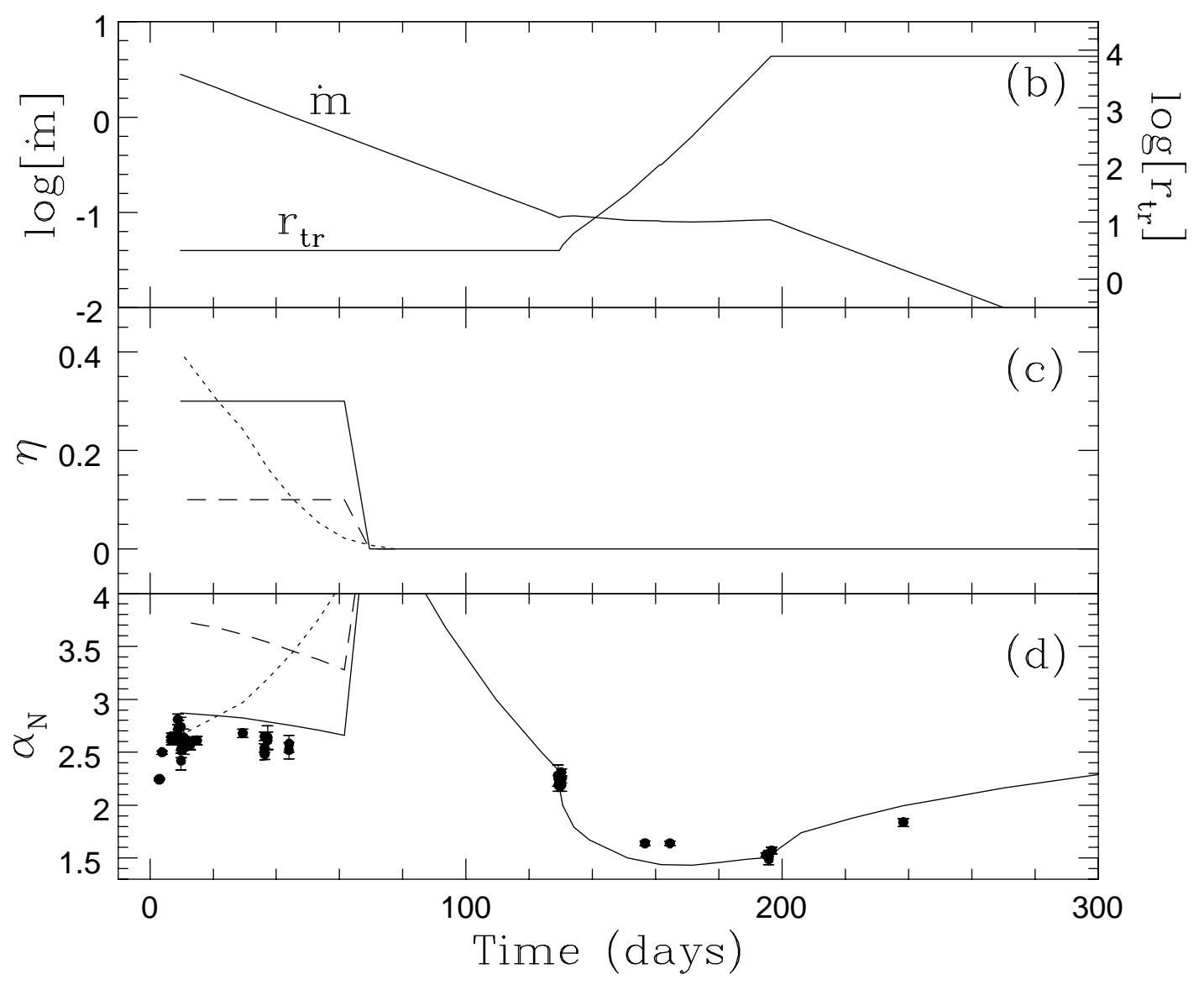

Fig. 12.- (b) Variation of $\dot{m}$ and $r_{t r}$ with time during the Nova Muscae outburst, according to the model described by equation (4-1). (c) Time variation of $\eta$ for the three models of the very high state: $\eta=0.3$ (solid line), $\eta=0.1$ (dashed line), variable $\eta$ (dotted line). (d) Time variation of the photon-index $\alpha_{N}$ of the hard power-law component of the spectrum. Filled points with error bars are the data from E94. The lines correspond to model results, with the spectral index computed in the range $10-20 \mathrm{keV}$ (the line types are as in panel c). 


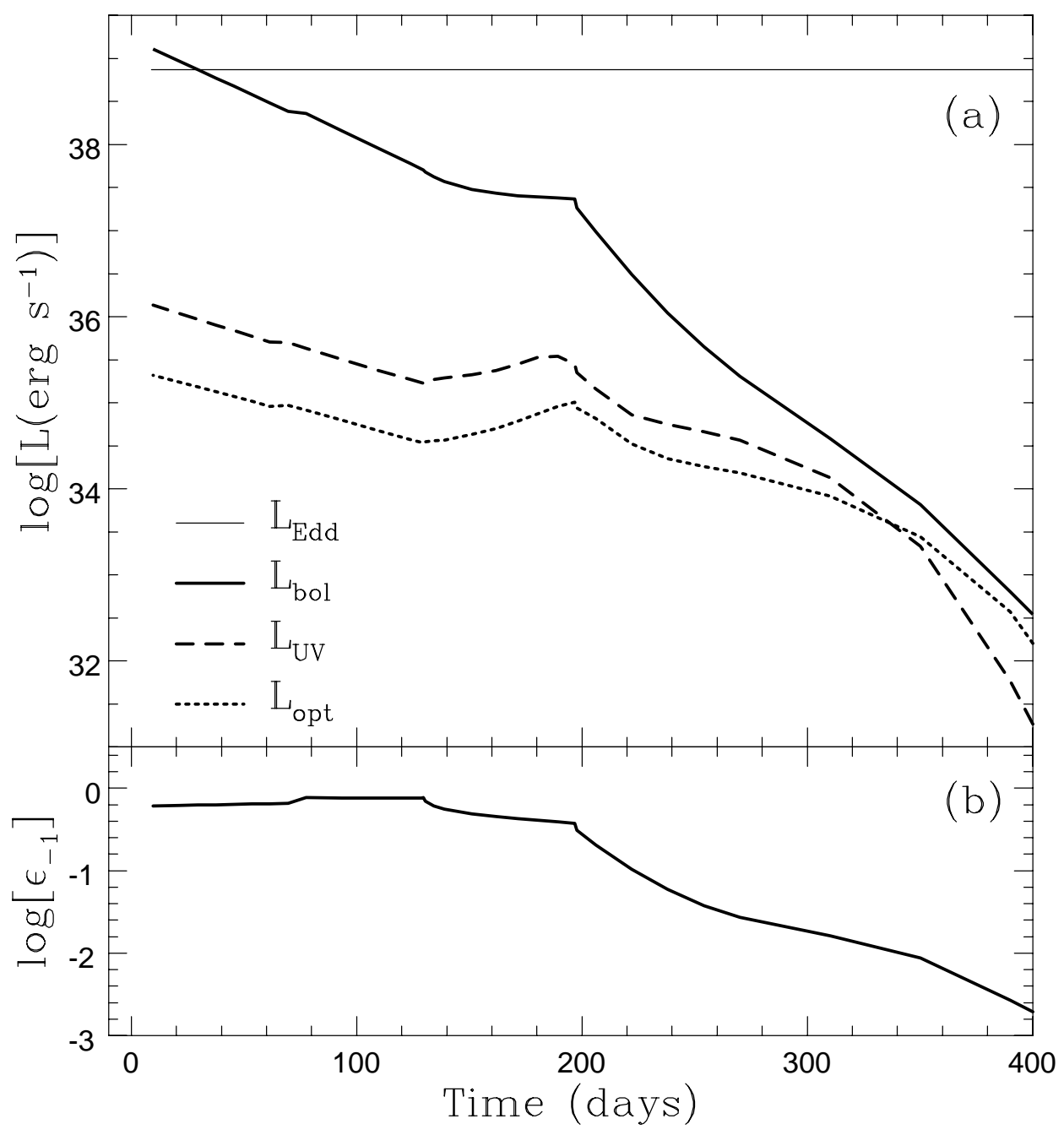

Fig. 13.- (a) The bolometric, optical $\left(10^{14.5}-10^{15} \mathrm{~Hz}\right)$ and UV $\left(10^{15}-10^{15.5} \mathrm{~Hz}\right)$ light curves of Nova Muscae, computed for the model with $\eta=0.3$ in the very high state. The relationship between Time, $\dot{m}$ and $r_{t r}$ is given by equation (4-1) and plotted in Figure 13. The horizontal line at the top is the Eddington luminosity for a $6 M_{\odot}$ accreting object. (b) Variation of the radiative efficiency of the accretion flow, $\epsilon_{-1}=L_{b o l} /\left(0.1 \dot{M} c^{2}\right)$, with time during the outburst. 


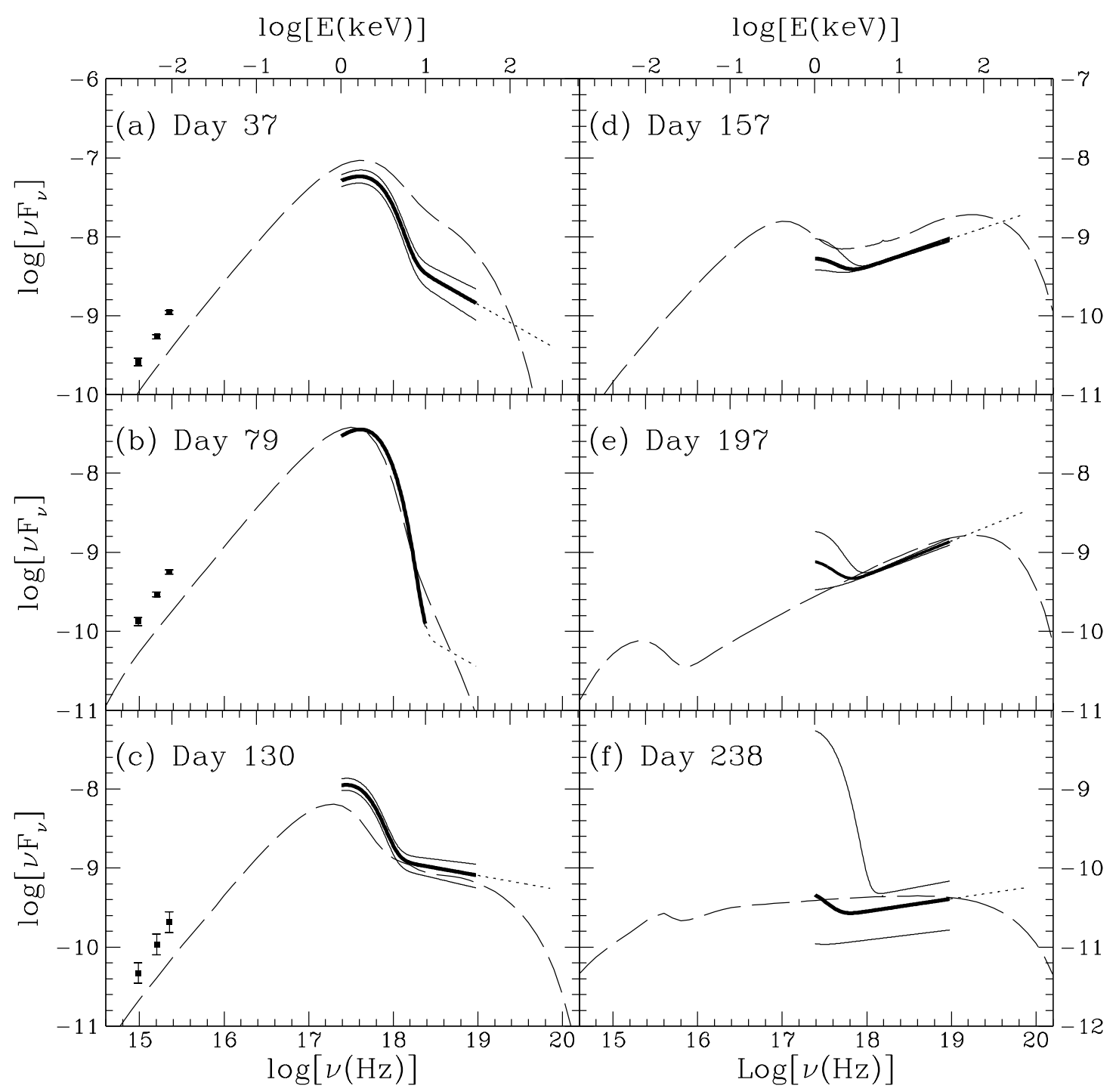

Fig. 14. - Spectra of Nova Muscae on six different days during the outburst, which correspond to the following entries in Table 2 of E94: 26, 33, 43, 44, 49, and 51. The heavy solid lines show the observed spectra reconstructed from the spectral fit parameters published by E94. The thin solid lines are 1- $\sigma$ upper and lower limits. The dotted lines in panels (a), (c)-(f) are extrapolations. The dotted line in panel (b) indicates that the powerlaw index of the hard end of the spectrum is highly uncertain. The UV data points are from Shrader \& Gonzalez-Riestra (1993). The dashed lines are model spectra corresponding to the values of $\dot{m}$ and $r_{t r}$ shown in Figure $12 \mathrm{~b}$ for each date. The model spectrum in panel (a) corresponds to the very high state with $\eta=0.3$. Panel (b) corresponds to the high state, panels (c) and (d) to the intermediate state, and panels (e) and (f) to the low state. 